ESAIM: M2AN 47 (2013) 1691-1712

DOI: $10.1051 / \mathrm{m} 2 \mathrm{an} / 2013082$
ESAIM: Mathematical Modelling and Numerical Analysis

www.esaim-m2an.org

\title{
LOWER AND UPPER BOUNDS FOR THE RAYLEIGH CONDUCTIVITY OF A PERFORATED PLATE
}

\author{
S. Laurens ${ }^{1}$, S. Tordeux ${ }^{2,3}$, A. Bendali ${ }^{4}$, M. Fares ${ }^{5}$ and P.R. Kotiuga ${ }^{6}$
}

\begin{abstract}
Lower and upper bounds for the Rayleigh conductivity of a perforation in a thick plate are usually derived from intuitive approximations and by physical reasoning. This paper addresses a mathematical justification of these approaches. As a byproduct of the rigorous handling of these issues, some improvements to previous bounds for axisymmetric holes are given as well as new estimates for tilted perforations. The main techniques are a proper use of the Dirichlet and Kelvin variational principles in the context of Beppo-Levi spaces. The derivations are validated by numerical experiments in $2 \mathrm{D}$ for the axisymmetric case as well as for the full three-dimensional problem.
\end{abstract}

Mathematics Subject Classification. 35Q35, 35J05, 35J25.

Received April 10, 2012. Revised February 22, 2013.

Published online October 11, 2013.

\section{INTRODUCTION}

\subsection{Effective impedance boundary conditions}

The Rayleigh conductivity is a key ingredient in the construction of effective conditions making it possible to easily account for the transmission and the reflection of an acoustic wave by a plate with small perforations. Actually, this parameter is involved when matching the far field, i.e., the overall behavior of the acoustic field near the plate in the context of the method of matched asymptotic expansions, and the near field which consists of a model for the fluid at the level of each perforation. This role has been mentioned in Rayleigh's seminal work [26] and remarkably worked out by Howe [10,12]. The parameter dealt with in this work is that introduced by Rayleigh [26]. It corresponds to assuming that the incompressible Euler equations is the model governing the fluid at the level of each perforation. As a result, it can be used as such when assuming that the acoustic equations remain valid even at the scale of the perforation [30] or incorporated in some other more elaborated

Keywords and phrases. Rayleigh conductivity, perforated plate, Kelvin principle, Dirichlet principle.

1 CERFACS - EMA, 42 avenue Gaspar Coriolis, 31100 Toulouse, France. sophie.laurens@insa-toulouse.fr

2 INRIA Bordeaux Sud-Ouest - Magique 3D

3 Université de Pau, LMA (UMR-CNRS 5142), avenue de l'Université, 64013 Pau, France. sebastien.tordeux@inria.fr

4 INSA-Mathematical Institute of Toulouse (UMR-CNRS 5219), 135 avenue de Rangueil, 31077 Toulouse, France. abderrahmane.bendali@insa-toulouse.fr

5 CERFACS - EMA, 42 avenue Gaspar Coriolis, 31100 Toulouse, France. fares@cerfacs.fr

6 Boston University, Department of Electrical and Computer Engineering, 8 Saint Mary's Street, Boston MA, 02215, USA. prk@bu.edu 
expression for the Rayleigh conductivity as in Howe [10-12] or Melling models [19,21]. An attempt carried out in [18] to define the Rayleigh conductivity when the small scale model is set in terms of the linearized NavierStokes equations has also to be noted. It is worth mentioning that the model consisting in assuming that the acoustic equations remain valid everywhere is among all the others the only one where absorption of acoustic energy by the perforations is completely neglected.

However, numerically computing the Rayleigh conductivity is generally a non trivial task. This problem is three-dimensional in general. Its numerical solution thus may become rapidly hard to carry out due to the large size of the problem to be solved. Another difficulty concerns the fact that this problem is set on an unbounded domain with moreover a unbounded boundary so that its numerical solution should be tackled either by a boundary element method [28] or by deriving a truncating condition incorporating the behavior of the solution at infinity in an accurate way (see, e.g., $[8,15])$.

To overcome these difficulties, many authors proposed to approximate the Rayleigh conductivity by approximate formulas $[1,10,14,24]$, derived either analytically or by intuitive approaches. In these papers, it is hard to clearly distinguish among the approximations that are heuristic and the ones that can be rigorously established. The objective of this paper is first to provide a rigorous background for the derivation of such approximations. Furthermore, many studies were devoted to the comparison of these models with acoustic experiments $[4,14,16,22,23,25]$. However, due to the various approximations carried out to derive the models, it is almost impossible to distinguish the modeling errors coming from the approximate estimates of the Rayleigh conductivity from those induced by the choice of a specific model. Another objective of this paper is to make this point as clear as possible.

\subsection{Definition of the Rayleigh conductivity}

Let $\mathrm{P}$ be a straight infinite plate of finite thickness $h>0$ with one isolated perforation $\Omega$ as depicted in Figure 1. Thus, the plate coincides with

$$
\mathrm{P}=\left\{\mathbf{x} \in \mathbb{R}^{2} \times\left[s_{-}, s_{+}\right] \text {and } \mathbf{x} \notin \Omega\right\},
$$

with $s_{+}-s_{-}=h, \Omega \subset \mathbb{R}^{2} \times\left[s_{-}, s_{+}\right]$a simply connected bounded domain and $x_{3}$ varying in $\left[s_{-}, s_{+}\right]$. The domain filled by the fluid is $\mathrm{D}=\mathbb{R}^{3} \backslash \mathrm{P}$, and can hence be seen as the domain admitting $\Omega$ and the following two half-spaces

$$
\mathrm{D}_{+}=\left\{\mathbf{x} \in \mathrm{D} \mid x_{3}>s_{+}\right\} \text {and } \mathrm{D}_{-}=\left\{\mathbf{x} \in \mathrm{D} \mid x_{3}<s_{-}\right\},
$$

as a non overlapping decomposition. We denote by $\Gamma_{+}$and $\Gamma_{-}$the lower and upper apertures of the perforation $\Omega$, i.e.,

$$
\Gamma_{ \pm}=\left\{\mathbf{x} \in \partial \Omega \mid x_{3}=s_{ \pm}\right\} .
$$

The lateral part of the boundary of $\Omega$ is $\Sigma=\bar{\Omega} \cap \overline{\mathrm{P}}$, hence $\partial \Omega$ can be expressed also in terms of its non overlapping decomposition in $\Gamma_{-}, \Gamma_{+}$and $\Sigma$. In most models, the difference of pressure $P^{+}(t)-P^{-}(t)$ from one to the other side of the perforation is proportional to the time derivative of the volume flux $Q=\int_{\Gamma_{-}} v_{3} \mathrm{~d} s$ through the aperture counted positively along the direction of the $x_{3}$-axis. Here, $P^{+}(t)$ and $P^{-}(t)$ are the limiting values for the pressure of the far field and are taken as values at infinite distance of the near field in the matching procedure. It is therefore usual to characterize the corresponding ratio at the small scale level as the Rayleigh conductivity $K_{R}$ of the aperture [12], p. 354

$$
K_{R}=\frac{\rho_{0} \partial_{t} Q(t)}{P^{-}(t)-P^{+}(t)},
$$

with $\rho_{0}$ the density of the fluid at rest, $v_{3}$ the component along $x_{3}$-axis of the velocity $\mathbf{v}, P^{+}(t)$ and $P^{-}(t)$ the pressures on the upper and lower side of the perforated plate. The Rayleigh conductivity has the dimension of length, and equation (1.3) is the analogue of Ohm's law. 


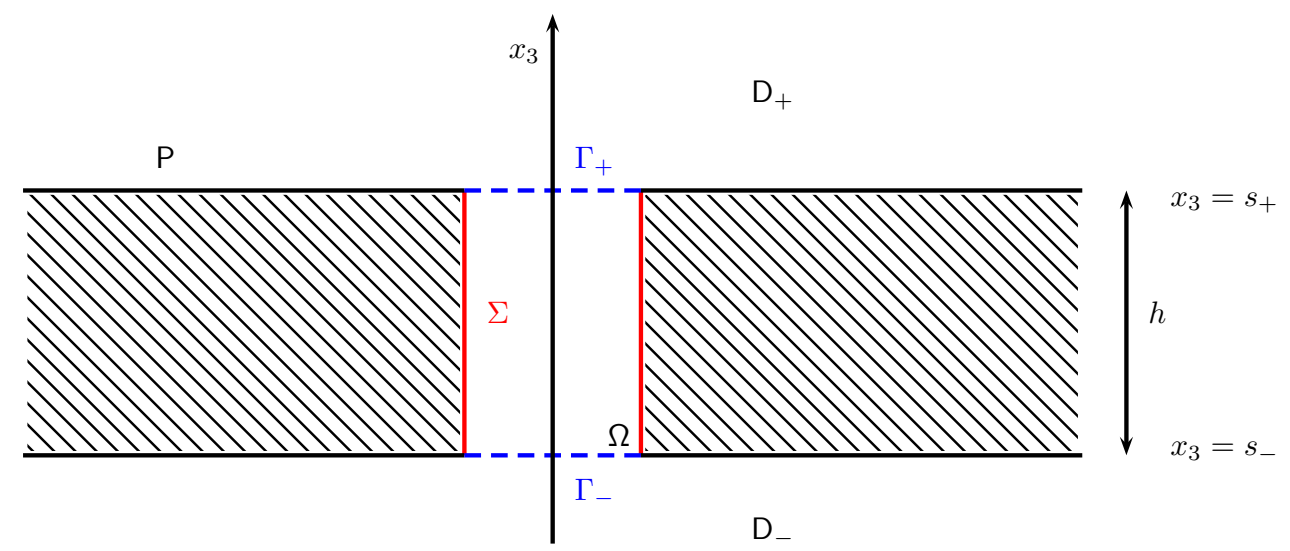

Figure 1: Schematic representation of the plate $\mathrm{P}$ (hatched) and the open aperture $\Omega$. The dashed lines represent the fictitious boundaries $\Gamma_{+}$and $\Gamma_{-}$of $\Omega$, while the straight line is its interior boundary $\Sigma$.

For the case of incompressible Euler equations considered here as said above, the governing equations can be expressed in terms of the following boundary-value problem set in the unbounded domain $\mathrm{D}(c f$. . [12]):

$$
\begin{cases}\rho_{0} \partial_{t} \mathbf{v}(\mathbf{x}, t)+\nabla p(\mathbf{x}, t)=0, & \text { in } \mathrm{D}, \\ \nabla \cdot \mathbf{v}(\mathbf{x}, t)=0, & \text { in } \mathrm{D}, \\ \partial_{\mathbf{n}} p(\mathbf{x}, t)=0, & \text { on } \partial \mathrm{D}, \\ \lim _{|\mathbf{x}| \rightarrow+\infty} p(\mathbf{x}, t)=P^{ \pm}(t), & \end{cases}
$$

yielding the Rayleigh conductivity as follows

$$
K_{R}=\frac{1}{P^{+}(t)-P^{-}(t)} \int_{\Gamma_{-}} \partial_{3} p(\mathbf{x}, t) \mathrm{d} s_{\mathbf{x}} .
$$

The incompressibility condition directly shows that the pressure satisfies the following boundary-value problem

$$
\begin{cases}\Delta p(\mathbf{x}, t)=0, & \text { in } \mathrm{D}, \\ \partial_{\mathbf{n}} p(\mathbf{x}, t)=0, & \text { on } \partial \mathrm{D}, \\ \lim _{|x| \rightarrow+\infty} p(\mathbf{x}, t)=P^{ \pm}(t) & \end{cases}
$$

and therefore that it does not depend on the initial conditions. Actually, setting

$$
u(\mathbf{x})=\frac{p(\mathbf{x}, t)-\left(P^{+}(t)+P^{-}(t)\right) / 2}{P^{+}(t)-P^{-}(t)}
$$

readily shows that $K_{R}$ is a constant depending only on the geometry of the perforation which can be defined by

$$
K_{R}=\int_{\Gamma_{-}} \partial_{x_{3}} u \mathrm{~d} s
$$

and

$$
\begin{cases}\Delta u(\mathbf{x})=0 & \text { in } \mathrm{D} \\ \partial_{\mathbf{n}} u(\mathbf{x})=0 & \text { on } \partial \mathrm{D} \\ \lim _{|x| \rightarrow+\infty} u(\mathbf{x})= \pm 1 / 2 & \text { on } \mathrm{D}_{ \pm} .\end{cases}
$$




\subsection{Contents of the paper}

The present paper is organized as follows. In Section 2, some links between Beppo Levi function spaces and the space of functions with square integrable gradients are brought out and made clearer. In Section 3, we introduce the Dirichlet and Kelvin dual principles which are the main tools for deriving bounds of the Rayleigh conductivity. Section 4 is devoted to the derivation of analytical expressions for the bounds for cylindrical, conical and tilted apertures. In Section 5 , the obtained formulas are illustrated by numerical experiments. The computations are carried out for an axi-symmetric perforation by solving a two-dimensional problem and by means of the three-dimensional boundary element code CESC of CERFACS for a perforation of general shape.

\section{FunCtions With SQUARE INTEGRABLE GRADIENT}

The classical functional setting for solving Laplace problems posed on three-dimensional unbounded domains is the Beppo-Levi space [2], the space of functions that "weakly tend to zero at infinity" whose gradient is square integrable. Unfortunately, this space is not adapted for the solution of problem (1.7) whose solution has to be sought in

$$
\mathrm{H}=\left\{v \in H_{\mathrm{loc}}^{1}(\overline{\mathrm{D}}) \mid \nabla v \in L^{2}(\mathrm{D})\right\},
$$

where $H_{\text {loc }}^{1}(\overline{\mathrm{D}})$ is the space of functions $v$ defined almost everywhere in $\mathrm{D}$ such that $\varphi v \in H^{1}(\mathrm{D})$ for all $\varphi \in \mathcal{D}\left(\mathbb{R}^{3}\right)$. In this section, we make clearer the relation between $\mathrm{H}$ and the Beppo-Levi space in dimension 3. To prove that problem (1.7) is well-posed, we first derive a Green formula involving functions in $\mathrm{H}$ and vector fields in

$$
W=\left\{\mathbf{q} \in \mathbf{L}^{2}(\mathrm{D}) \mid \operatorname{div} \mathbf{q}=0 \text { in } \mathrm{D} \text { and } \mathbf{q} \cdot \mathbf{n}=0 \text { on } \partial \mathrm{D}\right\} .
$$

Domain $A$ refers either to $D, D_{-}$or $D_{+}$. The Beppo-Levi space $B L(A)$ is defined as the closure of $\mathcal{D}(\bar{A})=$ $\left\{\left.u\right|_{\mathrm{A}} \mid u \in \mathcal{D}\left(\mathbb{R}^{3}\right)\right\}$ with respect to the $H^{1}$ semi-norm, a norm when restricted to $\mathcal{D}(\overline{\mathrm{A}})[7]$

$$
|v|_{\mathrm{BL}(\mathrm{A})}=\left(\int_{\mathrm{A}}|\nabla v(\mathbf{x})|^{2} \mathrm{~d} \mathbf{x}\right)^{\frac{1}{2}} .
$$

It is shown in [9] that $\mathrm{BL}(\mathrm{A})$ can be characterized as a Hilbert space and coincides with the weighted Sobolev space

$$
\mathrm{BL}(\mathrm{A})=\left\{v \in H_{\mathrm{loc}}^{1}(\mathrm{~A}) \mid \nabla v \in L^{2}(\mathrm{~A}) \text { and } \frac{v}{\left(1+|\mathbf{x}|^{2}\right)^{\frac{1}{2}}} \in L^{2}(\mathrm{~A})\right\} .
$$

The following proposition collects some useful properties of functions with square integrable gradients.

Proposition 2.1. Let $v \in \mathrm{H}$.

(i) The mean values of $v$ respectively on the hemisphere $\Gamma_{+}^{R}=\left\{\mathbf{x} \in \mathbb{R}^{3} \mid x_{3}>s_{+}\right.$and $\left.\left|\mathbf{x}-\mathbf{c}_{+}\right|=R\right\}$ and $\Gamma_{-}^{R}=\left\{\mathbf{x} \in \mathbb{R}^{3} \mid x_{3}<s_{-}\right.$and $\left.\left|\mathbf{x}-\mathbf{c}_{-}\right|=R\right\}$, with $\mathbf{c}_{ \pm}=\left(0,0, s_{ \pm}\right)$, converge as $R \rightarrow+\infty$, i.e.,

$$
v_{ \pm}:=\lim _{R \rightarrow+\infty} \frac{1}{2 \pi R^{2}} \int_{\Gamma_{ \pm}^{R}} v(\mathbf{x}) \mathrm{d} s_{\mathbf{x}} .
$$

(ii) The restrictions of $v$ to $\mathrm{D}_{-}$and $\mathrm{D}_{+}$satisfy $\left.v\right|_{\mathrm{D}_{ \pm}}-v_{ \pm} \in \mathrm{BL}\left(\mathrm{D}_{ \pm}\right)$.

(iii) The usual Beppo-Levi space $\mathrm{BL}(\mathrm{D})$ can be characterized as $\mathrm{BL}(\mathrm{D})=\left\{v \in \mathrm{H} \mid v_{-}=0\right.$ and $\left.v_{+}=0\right\}$.

(iv) The space $\mathrm{H}$ is a Hilbert space when equipped with the inner product

$$
(u, v)_{\mathrm{H}}=\int_{\mathrm{D}} \nabla u(\mathbf{x}) \cdot \nabla v(\mathbf{x}) \mathrm{d} \mathbf{x}+u_{+} v_{+}+u_{-} v_{-} .
$$


Proof. To prove point (i), we start by denoting $\left(r_{+}, \theta_{+}, \varphi_{+}\right)$the spherical coordinates with origin at $\mathbf{s}_{+}=\left(0,0, s_{+}\right)$

$$
x_{1}=r_{+} \sin \theta_{+} \cos \varphi_{+}, \quad x_{2}=r_{+} \sin \theta_{+} \sin \varphi_{+} \quad \text { and } \quad x_{3}=s_{+}+r_{+} \cos \theta_{+},
$$

and the associated metrics

$$
\mathrm{d} \sigma_{\mathbf{x}}=\sin \theta_{+} \mathrm{d} \theta_{+} \mathrm{d} \varphi_{+} \quad \text { and } \quad \mathrm{d} v_{\mathbf{x}}=\sin \theta_{+} \mathrm{d} \theta_{+} \mathrm{d} \varphi_{+} \mathrm{d} r_{+} .
$$

We denote by $\bar{v}(R)$ the mean value of $v$ over the half-sphere $\Gamma_{+}^{R}$

$$
\bar{v}(R)=\frac{1}{2 \pi} \int_{\mathrm{S}_{+}} v(\mathbf{x}) \mathrm{d} \sigma_{\mathbf{x}}=\frac{1}{2 \pi} \int_{0}^{\pi / 2} \int_{0}^{2 \pi} v\left(R, \theta_{+}, \varphi_{+}\right) \sin \theta_{+} \mathrm{d} \theta_{+} \mathrm{d} \varphi_{+} .
$$

Let us prove that $\bar{v}(R)$ satisfies the Cauchy criterion for convergence as $R$ tends to infinity. We have

$$
\left|\bar{v}\left(R^{\prime}\right)-\bar{v}(R)\right|=\frac{1}{2 \pi}\left|\int_{\mathrm{S}_{+}}\left(v\left(R^{\prime}, \theta_{+}, \varphi_{+}\right)-v\left(R, \theta_{+}, \varphi_{+}\right)\right) \mathrm{d} \sigma_{\mathbf{x}}\right| \leq \frac{1}{2 \pi}\left|\int_{\mathrm{S}_{+} \times\left[R, R^{\prime}\right]} \partial_{r_{+}} v\left(r_{+}, \theta_{+}, \varphi_{+}\right) \mathrm{d} v_{\mathbf{x}}\right|,
$$

with $\mathbf{S}_{+}$the part of the unit sphere $\mathbf{S}$ such that $x_{3}>0$. Applying the Cauchy-Schwartz inequality, we get

$$
\left|\bar{v}\left(R^{\prime}\right)-\bar{v}(R)\right| \leq \frac{1}{2 \pi}\left(\int_{\mathrm{S}_{+} \times\left[R, R^{\prime}\right]}\left|\partial_{r_{+}} v\left(r_{+}, \theta_{+}, \varphi_{+}\right)\right|^{2} r_{+}^{2} \mathrm{~d} v_{\mathbf{x}}\right)^{1 / 2}\left(\int_{\mathrm{S}_{+} \times\left[R, R^{\prime}\right]} \frac{1}{r_{+}^{2}} \mathrm{~d} v_{\mathbf{x}}\right)^{1 / 2} .
$$

This hence establishes the existence of $v_{+}$since

$$
\left|\bar{v}\left(R^{\prime}\right)-\bar{v}(R)\right| \leq \frac{1}{\sqrt{2 \pi}}|\nabla v|_{L^{2}\left(\mathrm{D}_{+}\right)}\left|\frac{1}{R}-\frac{1}{R^{\prime}}\right| 1 / 2 \underset{R, \underset{R^{\prime} \rightarrow+\infty}{\longrightarrow}}{\longrightarrow} 0 .
$$

The convergence to $v_{-}$is obtained in exactly the same way.

Now, we carry out the proof of point (ii). In view of Definition (2.2) of $\mathrm{BL}\left(\mathrm{D}_{+}\right)$, it is sufficient to show that $\left(\left.v\right|_{\mathrm{D}_{+}}-v_{+}\right) / \sqrt{1+r_{+}^{2}} \in L^{2}\left(\mathrm{D}_{+}\right)$or equivalently $\left(\left.v\right|_{\mathrm{D}_{+}}-v_{+}\right) / \sqrt{1+r_{+}^{2}} \in L^{2}\left(\mathrm{D}_{+, 1}\right)$ with

$$
\mathrm{D}_{+, 1}=\left\{\mathrm{x} \in \mathrm{D}_{+} \mid r_{+}>1\right\}=\mathrm{S}_{+} \times[1,+\infty) .
$$

This amounts to prove that $\left(\left.v\right|_{\mathrm{D}_{+}}(\mathbf{x})-\bar{v}\left(r_{+}\right)\right) / \sqrt{1+r_{+}^{2}} \in L^{2}\left(\mathrm{D}_{+, 1}\right)$ and $\left(\bar{v}\left(r_{+}\right)-v_{+}\right) / \sqrt{1+r_{+}^{2}} \in L^{2}\left(\mathrm{D}_{+, 1}\right)$.

To prove that $\left(\left.v\right|_{\mathrm{D}_{+}}-\bar{v}\left(r_{+}\right)\right) / \sqrt{1+r_{+}^{2}} \in L^{2}\left(\mathrm{D}_{+, 1}\right)$, we observe that

$$
\left|\frac{\left.v\right|_{\mathrm{D}_{+}}-\bar{v}\left(r_{+}\right)}{\left(1+r_{+}^{2}\right)^{\frac{1}{2}}}\right|_{L^{2}\left(\mathrm{D}_{+, 1}\right)}^{2}=\int_{\mathrm{D}_{+, 1}} \frac{\left(\left.v\right|_{\mathrm{D}_{+}}-\bar{v}\left(r_{+}\right)\right)^{2}}{1+r_{+}^{2}} r_{+}^{2} \mathrm{~d} v_{\mathbf{x}} \leq \int_{1}^{\infty} \int_{\mathrm{S}_{+}}\left(v\left(r_{+}, \theta_{+}, \varphi_{+}\right)-\bar{v}\left(r_{+}\right)\right)^{2} \mathrm{~d} v_{\mathbf{x}} .
$$

Using Poincaré inequality on the unit sphere, we get

$$
\left|\frac{\left.v\right|_{\mathrm{D}_{+}}-\bar{v}\left(r_{+}\right)}{\left(1+r_{+}^{2}\right)^{\frac{1}{2}}}\right|_{L^{2}\left(\mathrm{D}_{+, 1}\right)}^{2} \leq C \int_{1}^{\infty} \int_{\mathrm{S}_{+}}\left(\left(\partial_{\theta_{+}} v\right)^{2}+\left(\frac{\partial_{\varphi_{+}} v}{\sin \theta_{+}}\right)^{2}\right) \mathrm{d} v_{\mathbf{x}} .
$$

Since $|\nabla v|^{2}=\left(\partial_{r_{+}} v\right)^{2}+\left(\frac{1}{r_{+}} \partial_{\theta_{+}} v\right)^{2}+\left(\frac{1}{r_{+} \sin \theta_{+}} \partial_{\varphi_{+}} v\right)^{2}$, we obtain

$$
\left|\frac{\left.v\right|_{\mathrm{D}_{+}}-\bar{v}\left(r_{+}\right)}{\left(1+r_{+}^{2}\right)^{\frac{1}{2}}}\right|_{L^{2}\left(\mathrm{D}_{+, 1}\right)}^{2} \leq C \int_{1}^{\infty} \int_{\mathrm{S}_{+}}|\nabla v|^{2} r_{+}^{2} \mathrm{~d} v_{\mathbf{x}} \leq C|\nabla v|_{L^{2}\left(\mathrm{D}_{+, 1}\right)}^{2}<+\infty .
$$


Then, we seek to prove that $\left(\bar{v}\left(r_{+}\right)-v_{+}\right) / \sqrt{1+r_{+}^{2}} \in L^{2}\left(\mathrm{~S}_{+}\right)$. Since

$$
\left|\frac{\bar{v}\left(r_{+}\right)-v_{+}}{\left(1+r_{+}^{2}\right)^{\frac{1}{2}}}\right|_{L^{2}\left(\mathrm{D}_{+, 1}\right)}^{2} \leq \int_{1}^{\infty} \int_{\mathrm{S}_{+}} \frac{\left(\bar{v}\left(r_{+}\right)-v_{+}\right)^{2}}{1+r_{+}^{2}} r_{+}^{2} \mathrm{~d} v_{\mathbf{x}}
$$

taking $R=r_{+}$and letting $R^{\prime}$ tend to infinity in equation (2.5), we get

$$
\left|\bar{v}\left(r_{+}\right)-v_{+}\right| \leq \frac{1}{\sqrt{2 \pi}}|\nabla v|_{L^{2}\left(\mathrm{D}_{+}\right)} \frac{1}{r_{+}}
$$

and thus

$$
\left|\frac{\bar{v}\left(r_{+}\right)-v_{+}}{\left(1+r_{+}^{2}\right)^{\frac{1}{2}}}\right|_{L^{2}\left(\mathrm{D}_{+, 1}\right)}^{2} \leq \frac{1}{2 \pi}|\nabla v|_{L^{2}\left(\mathrm{D}_{+}\right)}^{2} \int_{1}^{\infty} \int_{\mathrm{S}_{+}} \frac{1}{1+r_{+}^{2}} \mathrm{~d} v_{\mathbf{x}} \leq \frac{\pi}{4}|\nabla v|_{L^{2}\left(\mathrm{D}_{+}\right)}^{2}<+\infty .
$$

Similarly, we have $\left.v\right|_{\mathrm{D}_{-}}-v_{-} \in \mathrm{BL}\left(\mathrm{D}_{-}\right)$.

To prove point (iii), we first establish that $\left\{v \in \mathrm{H} \mid v_{-}=0\right.$ and $\left.v_{+}=0\right\} \subset \mathrm{BL}(\mathrm{D})$. Let $v \in \mathrm{H}$ such that $v_{-}=v_{+}=0$. It follows from (ii) that $v \in \mathrm{BL}\left(\mathrm{D}_{-}\right)$and $v \in \mathrm{BL}\left(\mathrm{D}_{+}\right)$. Characterization (2.2) of $\mathrm{BL}$ in terms of weighted Sobolev space yields $v \in \mathrm{BL}(\mathrm{D})$.

Conversely, we prove that $\mathrm{BL}(\mathrm{D}) \subset\left\{v \in \mathrm{H} \mid v_{-}=0\right.$ and $\left.v_{+}=0\right\}$. If $u \in \mathrm{BL}(\mathrm{D})$, it follows from (ii) that $u-u_{+} \in \mathrm{BL}\left(\mathrm{D}_{+}\right)$and that $u-u_{-} \in \mathrm{BL}\left(\mathrm{D}_{-}\right)$. Therefore, $u_{ \pm}$belongs to $\mathrm{BL}\left(\mathrm{D}_{ \pm}\right)$. This is impossible except if $u_{ \pm}=0$ since $\int_{\mathrm{D}_{ \pm}} 1 /\left(1+|\mathbf{x}|^{2}\right) \mathrm{d} \mathbf{x}=+\infty$.

This brings us to point (iv). Clearly, $(u, v) \mapsto(u, v)_{\mathbf{H}}$ is a scalar product on $\mathbf{H}$. It first remains to establish that it is an Hilbert space. Let $\left(v_{n}\right)$ be a Cauchy sequence in $\mathrm{H}$. Denoting by $\xi_{ \pm}$two smooth cut-off functions such as $\xi_{ \pm}(\mathbf{x})=1$ in $D_{ \pm}$and $\xi_{ \pm}(\mathbf{x})=0$ in $\mathrm{D}_{\mp}$, we consider the function

$$
w_{n}=v_{n}-\left(v_{n}\right)_{+} \xi_{+}-\left(v_{n}\right)_{-} \xi_{-},
$$

that belongs to $\mathrm{BL}(\mathrm{D})$ as established in (ii). We first remark that $\left(w_{n}\right)$ is a Cauchy sequence of $\mathrm{BL}(\mathrm{D})$ equipped with its natural norm $|v|_{\mathrm{BL}(\mathrm{D})}=|\nabla v|_{L^{2}(\mathrm{D})}$ since $\nabla v_{n}$ is a Cauchy sequence in $L^{2}(\mathrm{D})$ and $\left(v_{n}\right)_{+},\left(v_{n}\right)_{-}$are convergent

$$
\left(v_{n}\right)_{+} \longrightarrow v_{+} \quad \text { and } \quad\left(v_{n}\right)_{-} \longrightarrow v_{-} \quad \text { with } v_{+}, v_{-} \in \mathbb{C} .
$$

It follows from the completeness of $\mathrm{BL}(\mathrm{D})$ that $w_{n}$ converges to $w \in \mathrm{BL}(\mathrm{D})$

$$
\nabla w_{n} \longrightarrow \nabla w \quad \text { in } L^{2}(\mathrm{D}) .
$$

The function $v=w+\xi_{+} \bar{v}_{+}+\xi_{-} \bar{v}_{-}$belongs to $\mathrm{H}$ and satisfies $v_{+}=\bar{v}_{+}, v_{-}=\bar{v}_{-}$. To complete the proof, it just remains to establish that $v_{n}$ converges to $v$ in $\mathrm{H}$

$$
\left\{\begin{aligned}
\left|\nabla\left(v_{n}-v\right)\right|_{L^{2}(\mathrm{D})} & \leq\left|\nabla\left(w_{n}-w\right)+\left(\left(v_{n}\right)_{+}-\bar{v}_{+}\right) \nabla \xi_{+}+\left(\left(v_{n}\right)_{-}-\bar{v}_{-}\right) \nabla \xi_{-}\right|_{L^{2}(\mathrm{D})} \\
& \leq\left|\nabla\left(w_{n}-w\right)\right|+\left|\left(\left(v_{n}\right)_{+}-\bar{v}_{+}\right) \nabla \xi_{+}\right|+\left|\left(\left(v_{n}\right)_{-}-\bar{v}_{-}\right) \nabla \xi_{-}\right|_{n \rightarrow+\infty} 0 . \\
\left|\left(v_{n}\right)_{ \pm}-v_{ \pm}\right| & =\left|\left(v_{n}\right)_{ \pm}-\bar{v}_{ \pm}\right| \rightarrow 0 \text { when } n \rightarrow \pm \infty
\end{aligned}\right.
$$

The following lemma establishes the above mentioned Green's formula involving a function $v$ in $\mathrm{H}$ and a vector field $\mathbf{q}$ in $\mathrm{W}$.

Lemma 2.2. For all $v \in \mathrm{H}$ and $q \in \mathrm{W}$, the following Green's formula holds true

$$
\int_{\mathrm{D}} \nabla v(\mathbf{x}) \cdot \mathbf{q}(\mathbf{x}) \mathrm{d} \mathbf{x}=\left(v_{+}-v_{-}\right) \int_{\Gamma_{ \pm}} q_{3}(\mathbf{x}) \mathrm{d} s_{\mathbf{x}} .
$$


Proof. Let $\mathrm{A}=\mathrm{D}, \mathrm{D}_{+}$or $\mathrm{D}_{-}$. For $w \in \mathrm{BL}(\mathrm{A})$ and $\mathbf{q} \in \mathrm{W}$ defined in equation (2.1), Green's formula yields [9]

$$
\int_{\mathbf{A}} \nabla w(\mathbf{x}) \cdot \mathbf{q}(\mathbf{x}) \mathrm{d} \mathbf{x}=\int_{\partial \mathbf{A}} w(\mathbf{x}) \mathbf{q}(\mathbf{x}) \cdot \mathbf{n} \mathrm{d} s_{\mathbf{x}}
$$

where $\mathrm{d} s_{\mathbf{x}}$ is the elemental area on $\partial \mathrm{A}$. The integral $I=\int_{\mathrm{D}} \nabla v(\mathbf{x}) \cdot \mathbf{q}(\mathbf{x}) \mathrm{d} \mathbf{x}$ is decomposed into three parts which are separately expressed by means of a Green's formula because $\left.v\right|_{\mathrm{D}_{ \pm}}-v_{ \pm} \in \mathrm{BL}\left(\mathrm{D}_{ \pm}\right)$.

$$
\left\{\begin{array}{c}
\int_{\mathrm{D}_{ \pm}} \nabla v(\mathbf{x}) \cdot \mathbf{q}(\mathbf{x}) \mathrm{d} \mathbf{x}=\int_{\mathrm{D}_{ \pm}} \nabla\left(v(\mathbf{x})-v_{ \pm}\right) \cdot \mathbf{q}(\mathbf{x}) \mathrm{d} \mathbf{x}=\mp \int_{\Gamma_{ \pm}}\left(v(\mathbf{x})-v_{ \pm}\right) q_{3}(\mathbf{x}) \mathrm{d} s_{\mathbf{x}} \\
\int_{\Omega} \nabla v(\mathbf{x}) \cdot \mathbf{q}(\mathbf{x}) \mathrm{d} \mathbf{x}=\int_{\Gamma_{+}} v(\mathbf{x}) q_{3}(\mathbf{x}) \mathrm{d} s_{\mathbf{x}}-\int_{\Gamma_{-}} v(\mathbf{x}) q_{3}(\mathbf{x}) \mathrm{d} s_{\mathbf{x}} .
\end{array}\right.
$$

Since $\int_{\Gamma_{-}} q_{3}(\mathbf{x}) \mathrm{d} s_{\mathbf{x}}=\int_{\Gamma_{+}} q_{3}(\mathbf{x}) \mathrm{d} s_{\mathbf{x}}$, we sum the above relations to obtain $I=\left(v_{+}-v_{-}\right) \int_{\Gamma_{-}} q_{3}(\mathbf{x}) \mathrm{d} s_{\mathbf{x}}$.

Lemma 2.3. There exists a unique solution $u \in \mathrm{H}$ to problem (1.7) characterized by $u \in \mathrm{H}_{1 / 2}$ and $\nabla u \in \mathrm{W}$, where $\mathrm{H}_{1 / 2}$ is the affine space

$$
\mathrm{H}_{1 / 2}=\left\{v \in \mathrm{H} \mid v_{+}=1 / 2 \text { and } v_{-}=-1 / 2\right\} .
$$

Proof. Let us first recall a similar result in $\mathrm{BL}(\mathrm{D})[9]$. For $f \in \mathcal{D}(\mathrm{D})$ and $g \in \mathcal{D}(\partial \mathrm{D})$, there exists one and only one $u \in \mathrm{BL}(\mathrm{D})$ such that

$$
\Delta u=f \text { in } \mathrm{D} \quad \text { and } \quad \partial_{\mathbf{n}} u=g \text { on } \partial \mathrm{D} .
$$

We first start establishing the uniqueness of the solution. If $u_{1} \in \mathrm{H}_{1 / 2}$ and $u_{2} \in \mathrm{H}_{1 / 2}$ are two solutions to (1.7), $w=u_{1}-u_{2}$ belongs to $\mathrm{BL}(\mathrm{D})$ and satisfies $\Delta w=0$ in $\mathrm{D}$ and $\partial_{\mathbf{n}} w=0$ on $\partial \mathrm{D}$. Property (2.9) directly yields that $w=0$.

We now move on to seeking a solution. Let us define $w \in \mathrm{BL}(\mathrm{D})$ by

$$
\Delta w=-\frac{\Delta \xi_{+}}{2}+\frac{\Delta \xi_{-}}{2} \text { and } \partial_{\mathbf{n}} w=-\frac{\partial_{\mathbf{n}} \xi_{+}}{2}+\frac{\partial_{\mathbf{n}} \xi_{-}}{2} .
$$

Function $u=w+\xi_{+} / 2-\xi_{-} / 2 \in \mathrm{H}_{1 / 2}$ and satisfies

$$
\Delta u(\mathbf{x})=0 \text { in } \mathrm{D} \text { and } \partial_{\mathbf{n}} u(\mathbf{x})=0 \text { on } \partial \mathrm{D} .
$$

It remains to prove that $\lim _{|x| \rightarrow+\infty} u(\mathbf{x})=1 / 2$ on $\mathrm{D}_{+}$and $\lim _{|x| \rightarrow+\infty} u(\mathbf{x})=-1 / 2$ on $\mathrm{D}_{-}$. This last point is easily obtained by a separation of variables.

\section{The Dirichlet And Kelvin PRinciples}

The Dirichlet and Kelvin principles ${ }^{7}$ are two variational principles, dual each to the other. This theory, which takes its roots in Lagrangian and Hamiltonian mechanics, is of great importance in several areas, particularly in mathematical modeling [6], Chapter 4, Section 9 to obtain lower and upper bounds for potential energies, in optimization [17] to derive the dual formulations, and in numerical analysis [27], Chapter 1 for assessing the validity or the accuracy of a numerical solution.

In this paper, these dual variational principles offer an ad-hoc framework to derive lower and upper bounds of the Rayleigh conductivity $K_{R}$.

\footnotetext{
${ }^{7}$ In the context of electrostatics, the Kelvin principle is also called the Thompson principle. William Thompson is the name at birth of Lord Kelvin.
} 
Proposition 3.1. The Rayleigh conductivity $K_{R}$ can be obtained equivalently in one of the following ways:

$$
\left\{\begin{array}{l}
\text { Dirichlet principle: } K_{R}=\min _{v \in \mathrm{H}_{1 / 2}} \mathrm{~J}_{1}(v) \text { with } \mathrm{J}_{1}(v)=\int_{\mathrm{D}}|\nabla v(\mathbf{x})|^{2} \mathrm{~d} \mathbf{x} \\
\text { Kelvin principle: } K_{R}=\max _{\mathbf{q} \in \mathrm{W}} \mathrm{J}_{2}(\mathbf{q}) \text { with } \mathrm{J}_{2}(\mathbf{q})=2 \int_{\Gamma_{-}} q_{3}(\mathbf{x}) \mathrm{d} s_{\mathbf{x}}-\int_{\mathrm{D}}|\mathbf{q}(\mathbf{x})|^{2} \mathrm{~d} \mathbf{x} .
\end{array}\right.
$$

Proof. Let $\mathrm{J}: \mathrm{H} \times L^{2}(\mathrm{D}) \longrightarrow \mathbb{R}^{+}$be the functional

$$
\mathrm{J}(v, \mathbf{q})=\int_{\mathrm{D}}|\mathbf{q}(\mathbf{x})-\nabla v(\mathbf{x})|^{2} \mathrm{~d} \mathbf{x} .
$$

We first prove that $(u, \nabla u)$ is the unique element of $\mathrm{H}_{1 / 2} \times \mathrm{W}$ where $\mathrm{J}$ reachs its minimum. Lemma 2.3 gives that the solution to problem (1.7) satisfies

$$
\mathrm{J}(u, \nabla u)=0, \quad u \in \mathrm{H}_{1 / 2}, \quad \nabla u \in \mathrm{W} .
$$

Thus $(u, \nabla u)$ is a point where $\mathrm{J}$ reaches its minimum on $\mathrm{H}_{1 / 2} \times \mathbf{W}$. On the other hand, every $(v, \mathbf{q}) \in \mathrm{H}_{1 / 2} \times \mathbf{W}$ satisfying $\mathbf{J}(v, \mathbf{q})=0$ is such that $\nabla v=\mathbf{q}$. Lemma 2.3 directly yields $v=u$ and $\mathbf{q}=\nabla u$. Consequently, the function $u$ is solution of (1.7) due to the fact that $\nabla u \in \mathrm{W}(c f .(2.1))$. The above claim is thus proved.

We now show that $J_{1}$ reaches its minimum at $u$ and $J_{2}$ its maximum at $\nabla u$. Lemma 2.2 gives that, for all $v \in \mathrm{H}_{1 / 2}$ and $\mathbf{q} \in \mathrm{W}$

$$
\int_{\mathrm{D}} \nabla v(\mathbf{x}) \cdot \mathbf{q}(\mathbf{x}) \mathrm{d} \mathbf{x}=\left(v_{+}-v_{-}\right) \int_{\Gamma_{-}} q_{3}(\mathbf{x}) \mathrm{d} s_{\mathbf{x}}=\int_{\Gamma_{-}} q_{3}(\mathbf{x}) \mathrm{d} s_{\mathbf{x}} .
$$

It follows that $\mathrm{J}(v, \mathbf{q})=\mathrm{J}_{1}(v)-\mathrm{J}_{2}(\mathbf{q})$ thus establishing that $\mathrm{J}_{1}(u) \leq \mathrm{J}_{1}(v)$ for all $v \in \mathrm{H}_{1 / 2}$ and $\mathrm{J}_{2}(\mathbf{q}) \leq \mathrm{J}_{2}(\nabla u)$ for all $\mathbf{q} \in \mathrm{W}$.

Finally we prove that $K_{R}=\mathrm{J}_{1}(u)=\mathrm{J}_{2}(\nabla u)$. Since $\mathrm{J}(u, \nabla u)=\mathrm{J}_{1}(u)-\mathrm{J}_{2}(\nabla u)=0$, we have $\mathrm{J}_{1}(u)=\mathrm{J}_{2}(\nabla u)$. To conclude, it remains to prove that $\mathrm{J}_{1}(u)=K_{R}$. Substituting $u$ for $v$ and $\nabla u$ for $\mathbf{q}$ in (3.2), we readily get $\mathrm{J}_{1}(u)=\int_{\mathrm{D}}|\nabla u(\mathbf{x})|^{2} \mathrm{~d} \mathbf{x}=\int_{\Gamma_{-}} \partial_{x_{3}} u(\mathbf{x}) \mathrm{d} s_{\mathbf{x}}=K_{R}$.

\section{Bounds for the Rayleigh Conductivity of some usual Perforations}

Proposition 3.1 is used to get upper and lower bounds of the Rayleigh conductivity for usual perforations. We consider axi-symmetric geometries, related to cylindrical and conical apertures, as well as perforations which give rise to a full three-dimensional problem.

\subsection{Cylindrical apertures}

Here, we consider a cylindrical perforation $\Omega=\left\{\left(x_{1}, x_{2}, x_{3}\right) \in \mathbb{R}^{3} \mid\left(x_{1}, x_{2}\right) \in \mathrm{S}_{R}\right.$ and $\left.x_{3} \in\left[s_{-}, s_{+}\right]\right\}$with circular section $\mathrm{S}_{R}=\left\{\left(x_{1}, x_{2}\right) \in \mathbb{R}^{2} \mid x_{1}^{2}+x_{2}^{2}<R^{2}\right\}$ (see Fig. 2 below).

Theorem 4.1. For a cylindrical perforation, we have the following bounds for its Rayleigh conductivity:

$$
K_{R, \mathrm{cyl}}^{-} \leq K_{R, \mathrm{cyl}} \leq K_{R, \mathrm{cyl}}^{+} \quad \text { with } K_{R, \mathrm{cyl}}^{-}=\frac{\pi R^{2}}{h+16 R / 3 \pi} \text { and } K_{R, \mathrm{cyl}}^{+}=\frac{\pi R^{2}}{h+\pi R / 2} .
$$

Proof. As established above, the Dirichlet principle allows us to obtain the upper bound

$$
K_{R, \text { cyl }} \leq \mathrm{J}_{1}(v) \text { for all } v \in \mathrm{H}_{1 / 2} .
$$




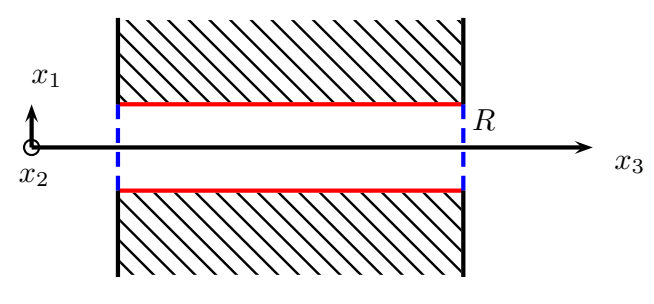

Figure 2: Cylindrical aperture of radius $R$.

We first consider the following problem

$$
\begin{cases}\Delta w_{R}(\mathbf{x})=0 & \text { for } x_{3} \neq 0 \\ \partial_{x_{3}} w_{R}\left(x_{1}, x_{2}, 0\right)=0 & \text { for } x_{1}^{2}+x_{2}^{2}>R^{2} \\ w_{R}\left(x_{1}, x_{2}, 0\right)=1 / 2 & \text { for } x_{1}^{2}+x_{2}^{2}<R^{2}\end{cases}
$$

whose solution $w_{R} \in \mathrm{BL}\left(\mathbb{R}^{3}\right)$ can be expressed analytically [29] Section 3.4, p. 72 by

$$
w_{R}(\mathbf{x})=\frac{1}{2 \pi^{2}} \int_{\mathrm{B}_{R}} \frac{1}{\sqrt{R^{2}-|\mathbf{y}|^{2}}} \frac{d s_{\mathbf{y}}}{|\mathbf{x}-\mathbf{y}|} \quad \text { for } x_{3} \neq 0
$$

with $\mathrm{B}_{R}=\left\{\left(y_{1}, y_{2}, y_{3}\right) \in \mathbb{R}^{3} \mid y_{1}^{2}+y_{2}^{2}<R^{2}\right.$ and $\left.y_{3}=0\right\}$. For $\alpha=\left(\alpha_{+}, \alpha_{-}\right) \in \mathbb{R}^{2}$ a pair of real numbers, we denote by $v_{\alpha}: \mathrm{D} \longrightarrow \mathbb{C}$ the function defined by

$$
v_{\alpha}(\mathbf{x})= \begin{cases}1 / 2-\left(1-2 \alpha_{+}\right) w_{R}\left(x_{1}, x_{2}, x_{3}-s_{+}\right) & \text {for } x_{3}>s_{+}, \\ \alpha_{-}+\left(\alpha_{+}-\alpha_{-}\right)\left(x_{3}-s_{-}\right) / h & \text { for } s_{-}<x_{3}<s_{+}, \\ -1 / 2+\left(1+2 \alpha_{-}\right) w_{R}\left(x_{1}, x_{2}, x_{3}-s_{-}\right) & \text {for } x_{3}<s_{-} .\end{cases}
$$

Proposition 2.1 gives that $v_{\alpha} \in \mathrm{H}_{1 / 2}$. Lemma A.1 in Appendix 5.3 then yields

$$
\int_{\mathrm{D}_{ \pm}}\left|\nabla w_{R}\left(x_{1}, x_{2}, x_{3}-s_{ \pm}\right)\right|^{2} \mathrm{~d} \mathbf{x}=R .
$$

Hence,

$$
\begin{aligned}
\mathrm{J}_{1}\left(v_{\alpha}\right) & =\int_{\mathrm{D}}\left|\nabla v_{\alpha}(\mathbf{x})\right|^{2} \mathrm{~d} \mathbf{x}=\int_{\mathrm{D}_{+}}\left|\nabla v_{\alpha}(\mathbf{x})\right|^{2} \mathrm{~d} \mathbf{x}+\int_{\Sigma}\left|\nabla v_{\alpha}(\mathbf{x})\right|^{2} \mathrm{~d} \mathbf{x}+\int_{\mathrm{D}_{-}}\left|\nabla v_{\alpha}(\mathbf{x})\right|^{2} \mathrm{~d} \mathbf{x} \\
& =R\left(1-2 \alpha_{+}\right)^{2}+\left(\alpha_{+}-\alpha_{-}\right)^{2} \pi R^{2} / h+R\left(1+2 \alpha_{-}\right)^{2} .
\end{aligned}
$$

Solving this elementary minimization problem, we get $\alpha_{+}=-\alpha_{-}=h /(\pi R+2 h)$ and $J_{1}\left(v_{\alpha}\right)=$ $\pi R^{2} /(h+\pi R / 2)$.

The lower bound is obtained from the Kelvin principle

$$
K_{R, \text { cyl }} \geq \mathrm{J}_{2}(\mathbf{q}) \text { for all } \mathbf{q} \text { in } \mathbf{W} .
$$

It is rather a classical matter that the following problems set respectively in $\left\{x_{3}>0\right\}$ and $\left\{x_{3}<0\right\}$

$$
\begin{cases}\Delta z_{R}(\mathbf{x})=0 & \text { for } x_{3} \neq 0 \\ \partial_{x_{3}} z_{R}\left(x_{1}, x_{2}, 0\right)=0 & \text { for } x_{1}^{2}+x_{2}^{2}>R^{2} \\ \partial_{x_{3}} z_{R}\left(x_{1}, x_{2}, 0_{ \pm}\right)= \pm 1 & \text { for } x_{1}^{2}+x_{2}^{2}<R^{2}\end{cases}
$$


admit one and only one solution in $\operatorname{BL}\left(\left\{x_{3}>0\right\}\right)$ and $\operatorname{BL}\left(\left\{x_{3}<0\right\}\right)$. This can be also obtained as a direct consequence of the characterization of these spaces given above in (2.2). The change of variable $x_{3} \rightarrow-x_{3}$ directly yields that $z_{R}$, piecewise defined from these solutions, is an even function of $x_{3}$ and so satisfies the transmission relations $\left[z_{R}\right] \mathrm{S}_{R}=\left(\left.z_{R}\right|_{z_{3}=0_{-}}-\left.z_{R}\right|_{z_{3}=0_{+}}\right) \mid \mathrm{s}_{R}=0$ and $\left[\partial_{x_{3}} z_{R}\right] \mathrm{S}_{R}=-2$. It can hence be expressed in terms of the following integral (see Sect. 3.4.1.3 of [28] p. 144)

$$
z_{R}(\mathbf{x})=-\frac{1}{2 \pi} \int_{\mathrm{B}_{R}} \frac{1}{|\mathbf{x}-\mathbf{y}|} \mathrm{d} s_{\mathbf{y}} \quad \text { for } x_{3} \neq 0
$$

This defines $\mathbf{q}_{\beta} \in \mathrm{W}$, up to a multiplicative constant $\beta \in \mathbb{R}$, such that

$$
\mathbf{q}_{\beta}(\mathbf{x})= \begin{cases}\beta \nabla z_{R}\left(x_{1}, x_{2}, x_{3}-s_{+}\right) / \pi R^{2} & \text { for } x_{3}>s_{+}, \\ \beta \mathbf{e}_{3} / \pi R^{2} & \text { for } s_{-}<x_{3}<s_{+}, \\ -\beta \nabla z_{R}\left(x_{1}, x_{2}, x_{3}-s_{-}\right) / \pi R^{2} & \text { for } x_{3}<s_{-}\end{cases}
$$

Using the non overlapping decomposition of $\mathrm{D}$ in $\mathrm{D}_{+}, \mathrm{D}_{-}$and $\Omega$, we can express $\mathrm{J}_{2}\left(\mathbf{q}_{\beta}\right)$ as follows

$$
\begin{aligned}
\mathrm{J}_{2}\left(\mathbf{q}_{\beta}\right) & =2 \int_{\Gamma_{-}} \mathbf{q}_{\beta}(\mathbf{x}) \cdot \mathbf{e}_{3} \mathrm{~d} s_{\mathbf{x}}-\int_{\mathrm{D}}\left|\mathbf{q}_{\beta}\right|^{2} \mathrm{~d} \mathbf{x}=2 \beta-\left(\int_{\mathrm{D}_{+}}\left|\mathbf{q}_{\beta}\right|^{2} \mathrm{~d} \mathbf{x}+\int_{\mathrm{D}_{-}}\left|\mathbf{q}_{\beta}\right|^{2} \mathrm{~d} \mathbf{x}+\int_{\Sigma}\left|\mathbf{q}_{\beta}\right|^{2} \mathrm{~d} \mathbf{x}\right) \\
& =2 \beta-\frac{\beta^{2}}{\pi^{2} R^{4}}\left(\int_{x_{3}>0}\left|\nabla z_{R}\right|^{2} \mathrm{~d} \mathbf{x}+\int_{x_{3}<0}\left|\nabla z_{R}\right|^{2} \mathrm{~d} \mathbf{x}+\pi R^{2} h\right)=2 \beta-\left(\frac{16 R}{3 \pi}+h\right) \frac{\beta^{2}}{\pi R^{2}}
\end{aligned}
$$

Integrals $\int_{x_{3}>0}\left|\nabla z_{R}\right|^{2} \mathrm{~d} \mathbf{x}=\int_{x_{3}<0}\left|\nabla z_{R}\right|^{2} \mathrm{~d} \mathbf{x}=8 R^{3} / 3$ are calculated in Appendix 5.3 (Lem. A.1). Maximizing this expression relatively to $\beta$, we obtain $\beta=\pi R^{2} /(h+16 R / 3 \pi)$, hence $\mathrm{J}_{2}\left(\mathbf{q}_{\beta}\right)=\pi R^{2} /(h+16 R / 3 \pi)$.

Remark 4.2. Estimate (4.1) was first stated by Rayleigh [26], then by Howe [12] Section 5.3 and was mostly obtained by physical reasoning. Here, the actual new feature of this approach is to establish it by means of rigorous arguments.

Remark 4.3. Since the two factors $16 / 3 \pi \simeq 1.6977$ and $\pi / 2 \simeq 1.5708$ involved in (4.1) do not greatly differ each from the other, this inequality leads to sharp bounds for the Rayleigh conductivity, as numerically illustrated below in Section 4.4.

Remark 4.4. Based on the approaches adopted by Rayleigh and Howe, in the proof of Theorem 4.1, we choose to use trial functions $v_{\alpha}$ and $\mathbf{q}_{\beta}$ which depend on $x_{3}$ only in the perforation. Estimate (4.1) can be improved by choosing more general trial functions. This way to proceed is a good compromise between the sharpness of the estimates and the simplicity of the calculations.

\subsection{Conical apertures}

We consider now a conical aperture of the following form

$$
\Omega=\left\{\mathbf{x} \in \mathbb{R}^{3} \mid\left(x_{1}, x_{2}\right) \in \mathrm{S}_{R\left(x_{3}\right)} \text { and } x_{3} \in\left[s_{-}, s_{+}\right]\right\}
$$

where the radius $R(s)$ is linearly varying from $R_{-}$to $R_{+}$(see Fig. 3 below)

$$
R(s)=R_{-}+\frac{s-s_{-}}{h}\left(R_{+}-R_{-}\right)=R_{-}+\left(s-s_{-}\right) \tan \varphi \quad \text { with } R_{+}>R_{-} .
$$




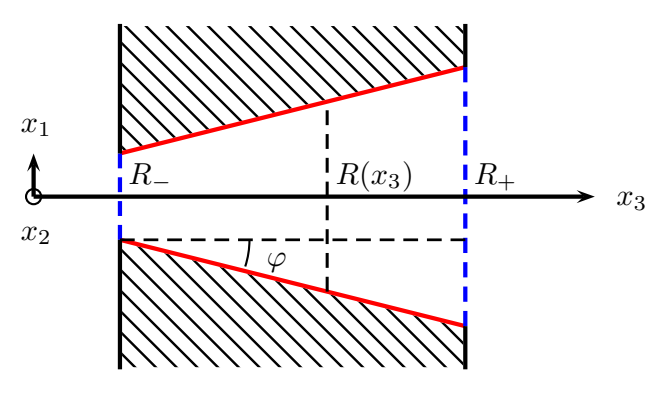

Figure 3: Conical aperture of radius $R\left(x_{3}\right)$.

Theorem 4.5. The following bounds for a conical aperture hold true

$$
K_{R, \mathrm{con}}^{-} \leq K_{R, \mathrm{con}} \leq K_{R, \mathrm{con}}^{+}
$$

with

$$
K_{R, \mathrm{con}}^{-}=\frac{\pi R_{-} R_{+}}{h+\frac{8}{3 \pi}\left(R_{-}+R_{+}\right)+\frac{1}{2 h}\left(R_{-}-R_{+}\right)^{2}} \quad \text { and } \quad K_{R, \mathrm{con}}^{+}=\frac{\pi R_{-} R_{+}}{h+\frac{\pi}{4}\left(R_{-}+R_{+}\right)},
$$

or equivalently

$$
K_{R, \mathrm{con}}^{-}=\frac{\pi R_{-}\left(R_{-}+h \tan \varphi\right)}{\frac{16 R_{-}}{3 \pi}+h\left(1+\frac{8}{3 \pi} \tan \varphi+\frac{1}{2} \tan ^{2} \varphi\right)} \quad \text { and } \quad K_{R, \mathrm{con}}^{+}=\frac{\pi R_{-}\left(R_{-}+h \tan \varphi\right)}{\frac{\pi R_{-}}{2}+h\left(1+\frac{\pi}{4} \tan \varphi\right)} .
$$

Proof. We follow the same approach as in Theorem 4.1.

To establish the upper bound, we consider $v_{\alpha} \in \mathrm{H}_{1 / 2}$ depending on two real constants $\alpha_{-}$and $\alpha_{+}$:

$$
v_{\alpha}(\mathbf{x})= \begin{cases}1 / 2-\left(1-2 \alpha_{+}\right) w_{R_{+}}\left(x_{1}, x_{2}, x_{3}-s_{+}\right) & \text {for } x_{3}>s_{+}, \\ \alpha_{-}+\left(\alpha_{+}-\alpha_{-}\right) \frac{\int_{s_{-}}^{x_{3}} \frac{d s}{\pi R^{2}(s)}}{\int_{s_{-}}^{s_{+}} \frac{d s}{\pi R^{2}(s)}} & \text { for } s_{-}<x_{3}<s_{+}, \\ -1 / 2+\left(1+2 \alpha_{-}\right) w_{R_{-}}\left(x_{1}, x_{2}, x_{3}-s_{-}\right) & \text {for } x_{3}<s_{-},\end{cases}
$$

where $w_{R_{ \pm}}$is the function $w_{R}$ defined in (4.3) with $R=R_{ \pm}$. Similarily as in the proof of Theorem 4.1, we get

$$
\begin{aligned}
\mathrm{J}_{1}\left(v_{\alpha}\right) & =\int_{\mathrm{D}_{-}}\left|\nabla v_{\alpha}(\mathbf{x})\right|^{2} \mathrm{~d} \mathbf{x}+\int_{\mathrm{D}_{+}}\left|\nabla v_{\alpha}(\mathbf{x})\right|^{2} \mathrm{~d} \mathbf{x}+\int_{\Sigma}\left|\nabla v_{\alpha}(\mathbf{x})\right|^{2} \mathrm{~d} \mathbf{x} \\
& =R_{-}\left(1+2 \alpha_{-}\right)^{2}+R_{+}\left(1-2 \alpha_{+}\right)^{2}+\left(\alpha_{+}-\alpha_{-}\right)^{2} \pi R_{+} R_{-} / h .
\end{aligned}
$$

As above, we minimize $J_{1}\left(v_{\alpha}\right)$ relatively to $\alpha_{ \pm}$to get

$$
\alpha_{ \pm}= \pm \frac{1}{2} \mp \frac{R_{\mp} \pi}{\pi\left(R_{+}+R_{-}\right)+4 h} \quad \text { and so } \quad J_{1}\left(v_{\alpha}\right)=\frac{4 \pi R_{+} R_{-}}{\pi\left(R_{+}+R_{-}\right)+4 h} .
$$


To obtain the lower bound, we consider the vector field $\mathbf{q}_{\beta} \in \mathrm{W}$, with $\beta \in \mathbb{R}$, given by

$$
\mathbf{q}_{\beta}(\mathbf{x})= \begin{cases}\frac{\beta}{\pi R_{+}^{2}} \nabla z_{R_{+}}\left(x_{1}, x_{2}, x_{3}-s_{+}\right) & \text {for } x_{3}>s_{+}, \\ \frac{\beta}{\pi R^{2}\left(x_{3}\right)}\left(\mathbf{e}_{3}+\frac{r}{R\left(x_{3}\right)} \frac{R_{+}-R_{-}}{h} \mathbf{e}_{r}\right) & \text { for } s_{-}<x_{3}<s_{+}, \\ -\frac{\beta}{\pi R_{-}^{2}} \nabla z_{R_{-}}\left(x_{1}, x_{2}, x_{3}-s_{-}\right) & \text {for } x_{3}<s_{-},\end{cases}
$$

with $z_{R}$ the function defined in (4.7). Proceeding as in the proof of Theorem 4.1, we get

$$
\mathrm{J}_{2}\left(\mathbf{q}_{\beta}\right)=2 \int_{\Gamma_{-}} \mathbf{q}_{\beta}(\mathbf{x}) \cdot \mathbf{e}_{3} \mathrm{~d} s_{\mathbf{x}}-\left(\int_{\mathrm{D}_{+}}\left|\mathbf{q}_{\beta}\right|^{2} \mathrm{~d} \mathbf{x}+\int_{\mathrm{D}_{-}}\left|\mathbf{q}_{\beta}\right|^{2} \mathrm{~d} \mathbf{x}+\int_{\Sigma}\left|\mathbf{q}_{\beta}\right|^{2} \mathrm{~d} \mathbf{x}\right) .
$$

As

$$
\int_{\Sigma}\left|\mathbf{q}_{\beta}\right|^{2} \mathrm{~d} \mathbf{x}=\frac{\beta^{2}}{\pi^{2}} \int_{s_{-}}^{s_{+}} \int_{0}^{2 \pi} \int_{0}^{R(s)} \frac{1}{R^{4}(s)}\left(1+\frac{r^{2}(s)}{R^{2}(s)}\left(\frac{R_{+}-R_{-}}{h}\right)^{2}\right) r \mathrm{~d} r \mathrm{~d} \theta \mathrm{d} s,
$$

it comes that

$$
\begin{aligned}
\mathrm{J}_{2}\left(\mathbf{q}_{\beta}\right)= & 2 \beta-\frac{\beta^{2}}{\pi^{2} R_{-}^{4}} \int_{x_{3}<0}\left|\nabla z_{R_{-}}\right|^{2} \mathrm{~d} \mathbf{x}-\frac{\beta^{2}}{\pi^{2} R_{+}^{4}} \int_{x_{3}>0}\left|\nabla z_{R_{+}}\right|^{2} \mathrm{~d} \mathbf{x}-\frac{\beta^{2} h}{\pi R_{-} R_{+}}\left(1+\frac{1}{2}\left(\frac{R_{+}-R_{-}}{h}\right)^{2}\right) \\
& =2 \beta-\beta^{2}\left(\frac{8}{3 \pi^{2}} \frac{R_{+}+R_{-}}{R_{+} R_{-}}+\frac{h}{\pi R_{-} R_{+}}\left(1+\frac{1}{2}\left(\frac{R_{+}-R_{-}}{h}\right)^{2}\right)\right) .
\end{aligned}
$$

It is then easy to maximize $\mathrm{J}_{2}\left(\mathbf{q}_{\beta}\right)$ relatively to $\beta$ to get

$$
\mathrm{J}_{2}\left(\mathbf{q}_{\beta}\right)=\pi R_{-} R_{+} /\left(h+\frac{8}{3 \pi}\left(R_{+}+R_{-}\right)+\frac{1}{2 h}\left(R_{+}-R_{-}\right)^{2}\right) .
$$

Remark 4.6. Bounds (4.10) of Theorem 4.5 have to be compared with the following ones obtained by Howe [12], p. 359, Section 5.3:

$$
\frac{h}{\pi R_{-} R_{+}}+\frac{1}{4}\left(\frac{1}{R_{-}}+\frac{1}{R_{+}}\right) \leq \frac{1}{K_{R}} \leq \frac{h}{\pi R_{-} R_{+}}+\frac{\left(R_{+}-R_{-}\right)^{2}}{\pi R_{-} R_{+} h}+\frac{8}{3 \pi^{2}}\left(\frac{1}{R_{-}}+\frac{1}{R_{+}}\right) .
$$

Putting them in the same form as (4.10), we get

$$
K_{R, \text { Howe }}^{-}=\frac{\pi R_{-} R_{+}}{h+\frac{8}{3 \pi}\left(R_{-}+R_{+}\right)+\frac{1}{h}\left(R_{-}-R_{+}\right)^{2}} \quad \text { and } \quad K_{R, \text { Howe }}^{+}=\frac{\pi R_{-} R_{+}}{h+\frac{\pi}{4}\left(R_{-}+R_{+}\right)} .
$$

There is a missed factor 2 in the last term in the denominator of the fraction expressing $K_{R \text {, Howe }}^{-}$which seems to be a mistyping.

\subsection{Tilted cylinder}

We are now investigating the case where the aperture is cylindrical and tilted with an angle $\theta$ :

$$
\Omega=\left\{\mathbf{x} \in \mathbb{R}^{3} \mid\left(\widehat{x}_{1}(\mathbf{x}), x_{2}\right) \in \mathrm{S}_{R} \text { and } x_{3} \in\left[s_{-}, s_{+}\right] \text {with } \widehat{x}_{1}(\mathbf{x})=x_{1}-\left(x_{3}-s_{-}\right) \tan \theta\right\} .
$$

The perforation was chosen so that its apertures in both sides of the plate are circular with a radius $\mathrm{R}$ (see Fig. 4 below). 


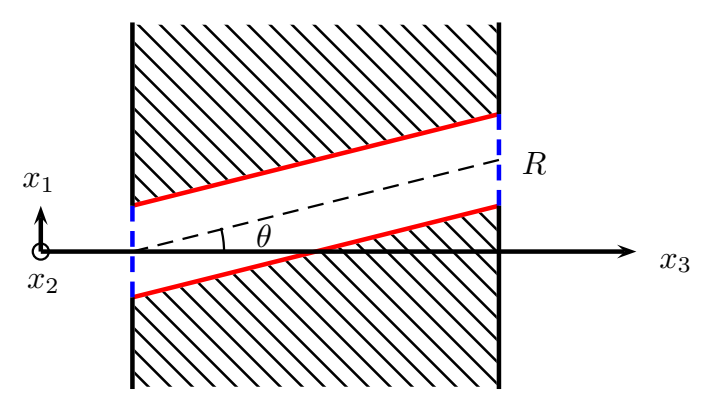

$$
x_{3}=s_{-} \quad x_{3}=s_{+}
$$

Figure 4: Cylindrical aperture tilted with angle $\theta$ with respect to the $x_{3}$ axis.

Theorem 4.7. The following bounds hold true for a tilted perforation

$$
K_{R, \text { inc }}^{-} \leq K_{R, \text { inc }} \leq K_{R, \text { inc }}^{+},
$$

with

$$
K_{R, \text { inc }}^{-}=\frac{\pi R^{2}}{\frac{16 R}{3 \pi}+\frac{h}{\cos ^{2} \theta}} \text { and } K_{R, \text { inc }}^{+}=\frac{\pi R^{2}}{\frac{\pi R}{2}+\frac{h}{\cos ^{2} \theta}\left(1+\frac{16 R}{3 \pi h} \sin ^{2} \theta\right)^{-1}}
$$

Proof. As above, we start with the upper bound. We consider $v_{\alpha}$ depending on a real constant $\alpha$ defined by:

$$
v_{\alpha}(\mathbf{x})=\left\{\begin{aligned}
1 / 2-(1-2 \alpha) w_{R}\left(x_{1}-h \tan \theta, x_{2}, x_{3}-s_{+}\right) & \\
\quad+\frac{\mu_{\alpha} \sin \theta}{h} t_{R}\left(x_{1}-h \tan \theta, x_{2}, x_{3}-s_{+}\right) & \text {for } x_{3}>s_{+}, \\
-\alpha+\frac{\mu_{\alpha}}{h}\left(\left(x_{3}-s_{-}\right) \cos \theta+x_{1} \sin \theta\right) & \text { for } s_{-}<x_{3}<s_{+}, \\
-1 / 2+(1-2 \alpha) w_{R}\left(x_{1}, x_{2}, x_{3}-s_{-}\right)+\frac{\mu_{\alpha} \sin \theta}{h} t_{R}\left(x_{1}, x_{2}, x_{3}-s_{-}\right) & \text {for } x_{3}<s_{-},
\end{aligned}\right.
$$

with $\mu_{\alpha}=2 \alpha \cos \theta, w_{R}$ given in (4.4) and $t_{R} \in \mathrm{BL}\left(\mathbb{R}^{3}\right)$ defined by

$$
\begin{cases}\Delta t_{R}(\mathbf{x})=0, & \text { for } x_{3} \neq 0 \\ \partial_{x_{3}} t_{R}\left(x_{1}, x_{2}, 0\right)=0, & \text { for } x_{1}^{2}+x_{2}^{2}>R^{2} \\ t_{R}\left(x_{1}, x_{2}, 0\right)=x_{1}, & \text { for } x_{1}^{2}+x_{2}^{2}<R^{2}\end{cases}
$$

To establish that $v_{\alpha}$ is in $\mathrm{H}_{1 / 2}$, it is enough to remark that it is continuous in an obvious way at $x_{3}=s_{-}$and that its limits at $x_{3}=s_{+}$from the left and from the right are respectively $-\alpha+2 \alpha \cos ^{2} \theta+\mu_{\alpha} x_{1} \sin \theta$ and $\alpha-2 \alpha \sin ^{2} \theta+\mu_{\alpha} x_{1} \sin \theta$.

Using Copson's method [29], Section 3.4, p. 72, an explicit integral expression can be given for $t_{R}$ :

$$
t_{R}(\mathbf{x})=\frac{2}{\pi^{2}} \int_{\mathrm{S}_{R}} \frac{y_{1}}{\sqrt{R^{2}-|\mathbf{y}|^{2}}} \frac{\mathrm{d} s_{\mathbf{y}}}{|\mathbf{x}-\mathbf{y}|} \quad \text { for } x_{3} \neq 0 .
$$

Similarly as in the Proof of Theorem 4.1

$$
\mathrm{J}_{1}\left(v_{\alpha}\right)=\int_{\mathrm{D}_{+}}\left|\nabla v_{\alpha}(\mathbf{x})\right|^{2} \mathrm{~d} \mathbf{x}+\int_{\Sigma}\left|\nabla v_{\alpha}(\mathbf{x})\right|^{2} \mathrm{~d} \mathbf{x}+\int_{\mathrm{D}_{-}}\left|\nabla v_{\alpha}(\mathbf{x})\right|^{2} \mathrm{~d} \mathbf{x} .
$$


Since $w_{R}$ is symmetric with respect to $x_{1}$ whereas $t_{R}$ is antisymmetric, we have

$$
\int_{x_{3}<0} \nabla t_{R}(\mathbf{x}) \cdot \nabla w_{R}(\mathbf{x}) \mathrm{d} \mathbf{x}=\int_{x_{3}>0} \nabla t_{R}(\mathbf{x}) \cdot \nabla w_{R}(\mathbf{x}) \mathrm{d} \mathbf{x}=0 .
$$

From calculations carried out in Appendix 5.3, it follows that:

$$
\mathrm{J}_{1}\left(v_{\alpha}\right)=2\left(R(1-2 \alpha)^{2}+\frac{\mu_{\alpha}^{2} \sin ^{2} \theta}{h^{2}} \frac{8 R^{3}}{3}\right)+\frac{\mu_{\alpha}^{2}}{h^{2}} \pi R^{2} h .
$$

As above, we maximize $\mathrm{J}_{1}\left(v_{\alpha}\right)$ relatively to $\alpha$ to obtain $\alpha=4 R /\left(8 R+4 \cos ^{2} \theta / h^{2}\left(16 R^{3} \sin ^{2} \theta / 3+\pi R^{2} h\right)\right)$,

$$
\mathrm{J}_{1}\left(v_{\alpha}\right)=\frac{\pi R^{2}\left(1+\frac{16 R}{3 \pi h} \sin ^{2} \theta\right)}{\frac{h}{\cos ^{2} \theta}+\frac{\pi R}{2}\left(1+\frac{16 R}{3 \pi h} \sin ^{2} \theta\right)} .
$$

We now establish the lower bound. Let us consider the vector field $\mathbf{q}_{\beta} \in \mathbf{W}$, with $\beta \in \mathbb{R}$, given by

$$
\mathbf{q}_{\beta}(\mathbf{x})= \begin{cases}\frac{\beta}{\pi R^{2}} \nabla z_{R}\left(x_{1}-h \tan \theta, x_{2}, x_{3}-s_{+}\right) & \text {for } x_{3}>s_{+}, \\ \frac{\beta}{\pi R^{2}}\left(\mathbf{e}_{3}+\tan \theta \mathbf{e}_{1}\right) & \text { for } s_{-}<x_{3}<s_{+}, \\ -\frac{\beta}{\pi R^{2}} \nabla z_{R}\left(x_{1}, x_{2}, x_{3}-s_{-}\right) & \text {for } x_{3}<s_{-} .\end{cases}
$$

with $z_{R}$ the function defined in (4.7). We have

$$
\mathrm{J}_{2}\left(\mathbf{q}_{\beta}\right)=2 \int_{\Gamma_{-}} \mathbf{q}_{\beta}(\mathbf{x}) \cdot \mathbf{e}_{3} \mathrm{~d} s_{\mathbf{x}}-\int_{\mathrm{D}_{+} \cup \mathrm{D}_{-} \cup \Sigma}\left|\mathbf{q}_{\beta}\right|^{2} \mathrm{~d} \mathbf{x}=2 \beta-\frac{\beta^{2}}{\pi^{2} R^{4}}\left(\frac{16 R^{3}}{3}+\pi R^{2} h\left(1+\tan ^{2} \theta\right)\right) .
$$

We maximize $\mathrm{J}_{2}\left(\mathbf{q}_{\beta}\right)$ relatively to $\beta$ to get $\mathrm{J}_{2}\left(\mathbf{q}_{\beta}\right)=\pi R^{2} /\left(h / \cos ^{2} \theta+16 R / 3 \pi\right)$.

Remark 4.8. Bounds (4.11) of Theorem 4.7 seem to be completely new. They have not been previously obtained even heuristically by physical reasoning. This result is especially important in applications related to combustion in turboengines. Indeed, in order to ensure the cooling of the combustion chamber, its wall is perforated by tilted holes. As seen above, the angle of the perforation axis with the plate has a great influence on its Rayleigh conductivity, and therefore, on its acoustic properties.

\subsection{Numerical validation of the previous bounds for the Rayleigh conductivity}

This section is devoted to a numerical validation of the above theoretical bounds obtained for the Rayleigh conductivity in Theorems 4.1, 4.5 and 4.7. A numerical approximation $K_{R, *}^{\text {num }}$ of the Rayleigh conductivity obtained by a direct numerical solution of problem (1.7) using the boundary element code CESC of CERFACS is compared with $K_{R, *}^{-}$and $K_{R, *}^{+}$for $h / R \in[0,10]$. The numerical procedure is detailled in Appendix 5.3 below. We have considered two angles $\varphi=5^{\circ}$ and $\varphi=10^{\circ}$ for a conical perforation and $\theta=15^{\circ}$ and $\theta=30^{\circ}$ for a tilted one. The results for straight cylindrical perforations, conical perforations, and tilted perforations are collected in Figures 5, 6 and 7. It is worth mentioning that $K_{R, *}^{-} \leq K_{R, *}^{\mathrm{num}} \leq K_{R, *}^{+}$in all cases.

\section{Approximate Rayleigh conductivity and CORRESPonding ERror}

\subsection{Approximate Rayleigh conductivity}

It is quite natural to take the mean value of the above lower and upper bounds for approximating the Rayleigh conductivity:

$$
K_{R}^{\mathrm{app}}=\frac{K_{R}^{+}+K_{R}^{-}}{2}
$$




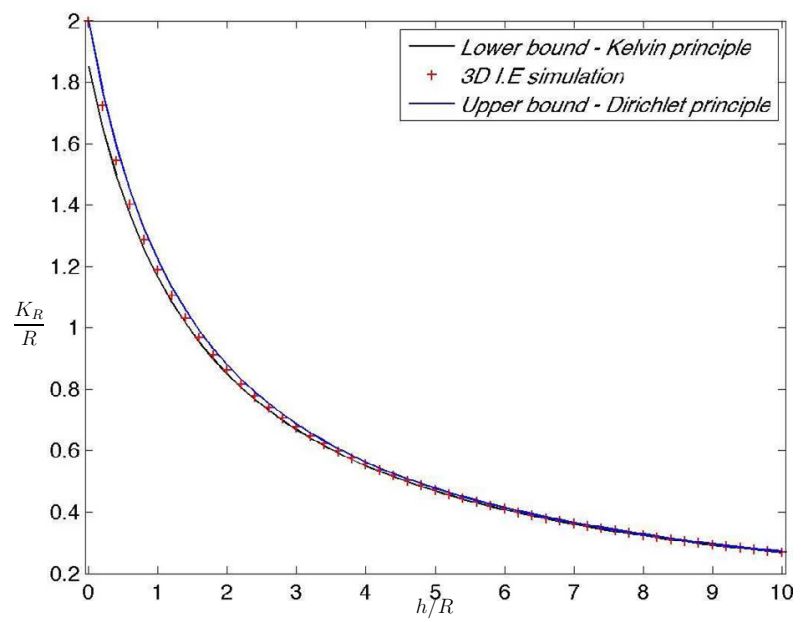

Figure 5: Lower and upper bounds and numerical approximation of the Rayleigh conductivity for a cylindrical perforation.
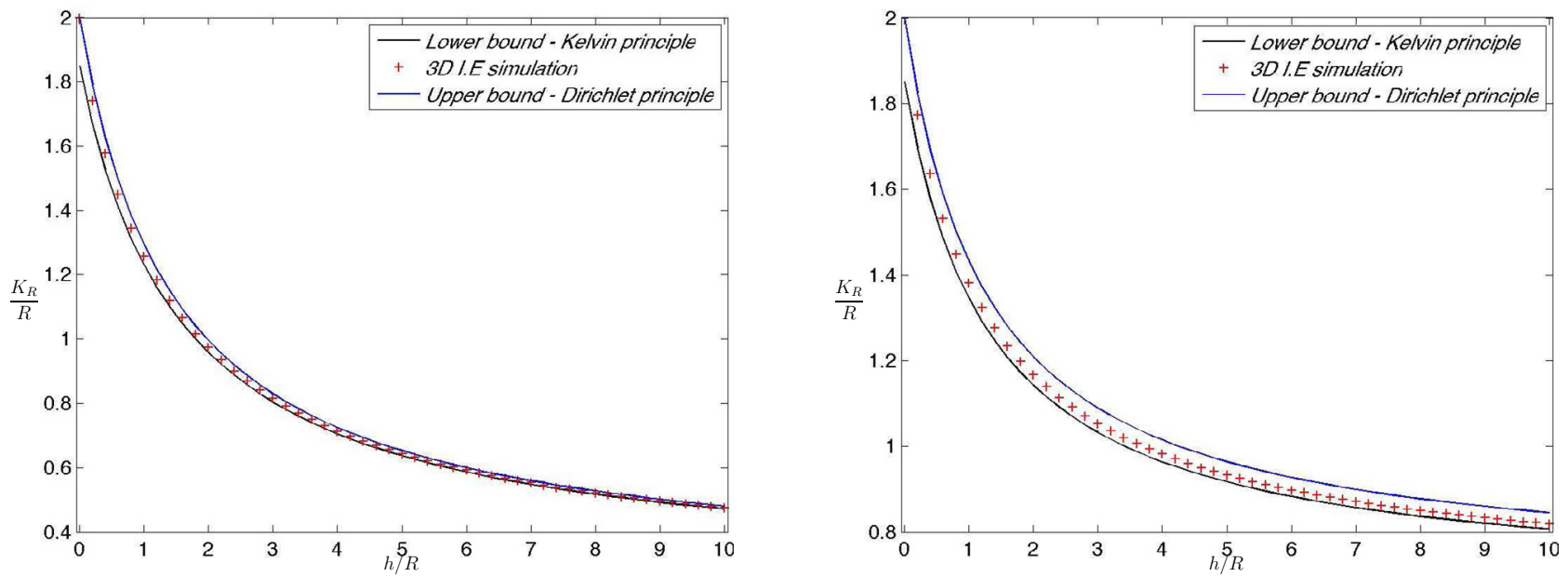

Figure 6: Lower and upper bounds and numerical approximation of the Rayleigh conductivity for a conical perforation with $\varphi=5^{\circ}$ and $\varphi=15^{\circ}$.

The relative error induced by this approximation is defined as

$$
\varepsilon=\left|\frac{K_{R}-K_{R}^{\mathrm{app}}}{K_{R}}\right| .
$$

A subscript as in $K_{R, \text { inc }}^{\text {app }}$ or $\varepsilon_{\text {cyl }}$ can be added for mentioning the case being considered.

\subsection{Theoretical bound on the error}

Since $K_{R}^{-} \leq K_{R} \leq K_{R}^{+}$, the error can be bounded as follows:

$$
\varepsilon \leq \frac{1}{2}\left(\frac{K_{R}^{+}-K_{R}^{-}}{K_{R}^{-}}\right)
$$



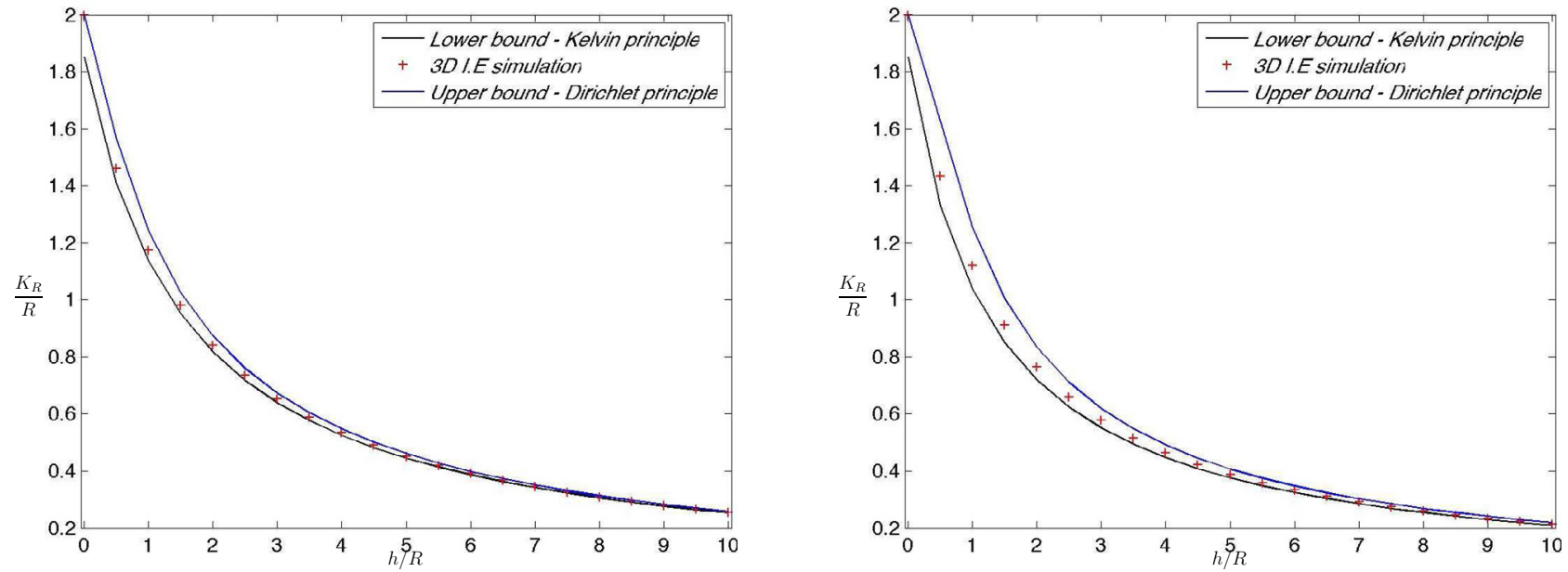

Figure 7: Lower and upper bounds and numerical approximation of the Rayleigh conductivity for a tilted perforation with $\theta=15^{\circ}$ and $\theta=30^{\circ}$.

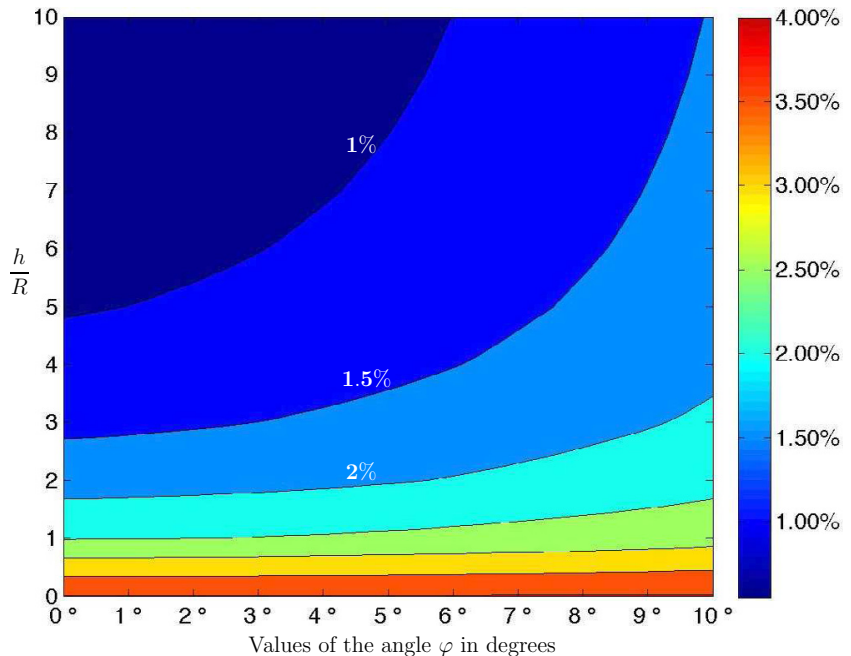

Figure 8: Upper bound of $\varepsilon_{\mathrm{con}}=\left|\frac{K_{R, \mathrm{con}}-K_{R, \mathrm{con}}^{\mathrm{app}}}{K_{R, \mathrm{con}}}\right|$.

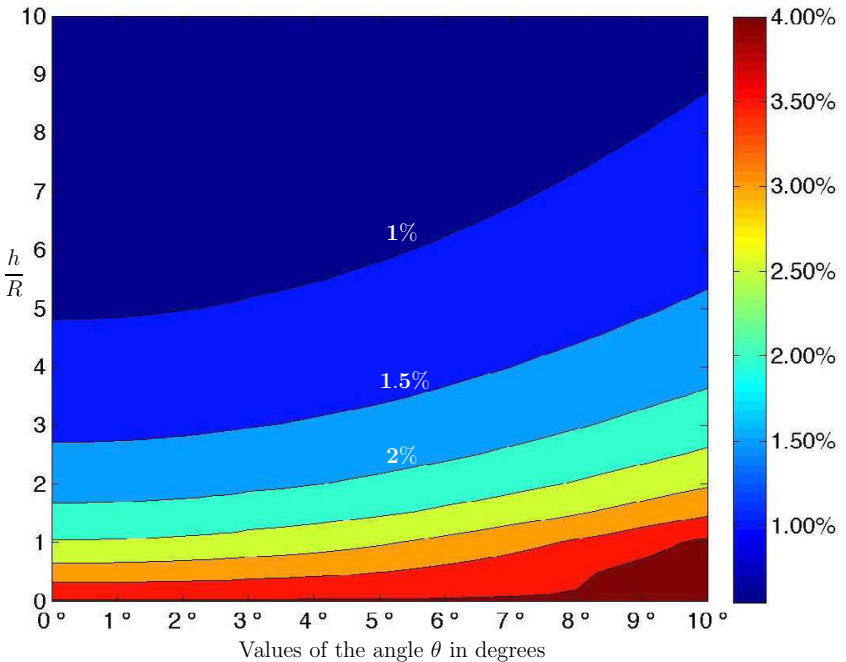

Figure 9: Upper bound of $\varepsilon_{\mathrm{inc}}=\left|\frac{K_{R, \mathrm{inc}}-K_{R, \text { inc }}^{\mathrm{app}}}{K_{R, \mathrm{inc}}}\right|$.

These inequalities are not optimal but give a good idea on the magnitude of the error. The right-hand sides of (5.2) are depicted in Figure 8 for a conical perforation and in Figure 9 for a tilted perforation. The results for a straight cylindrical perforation are reported in Figure 8 for $\varphi=0$ or in Figure 9 for $\theta=0$. Errors for $\varphi \leq 10^{\circ}$ and $\theta \leq 10^{\circ}$ are less than $5 \%$. For small values of $h$ the error is below $5 \%$. For larger values of $h$, the bound for the error is lower and mostly less than $2 \%$.

\subsection{Numerical study of the error}

In this section, we compare a numerical approximation $K_{R}^{\text {num }}$ of the Rayleigh conductivity to its analytical approximation $K_{R}^{\text {app }}$. For cylindrical and conical perforations, we have considered several configurations each of 


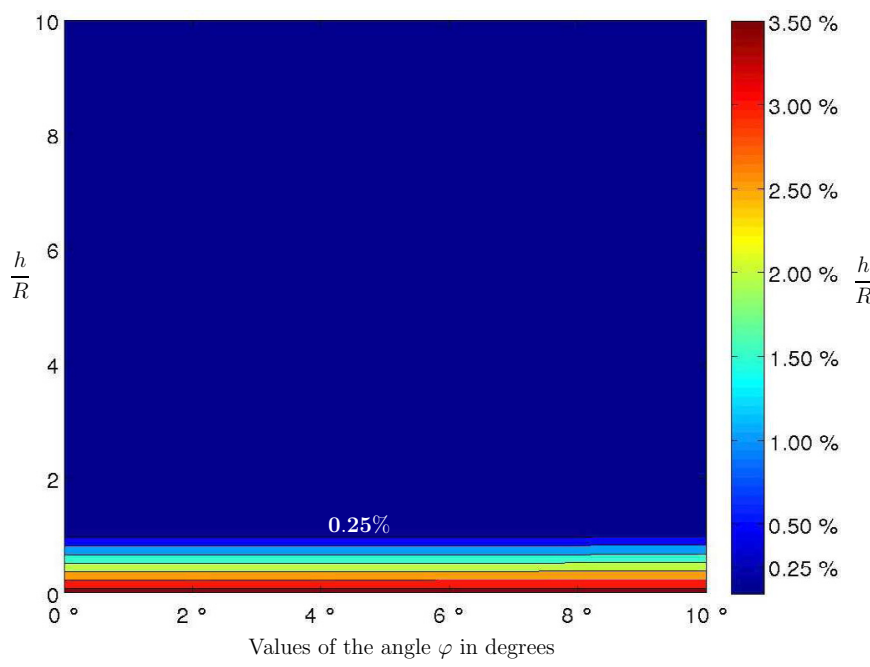

Figure 10: The error $\varepsilon_{\mathrm{con}}=\left|\frac{K_{R, \mathrm{con}}^{\mathrm{app}}-K_{R, \mathrm{con}}^{\mathrm{num}}}{K_{R, \mathrm{con}}^{\mathrm{num}}}\right|$.

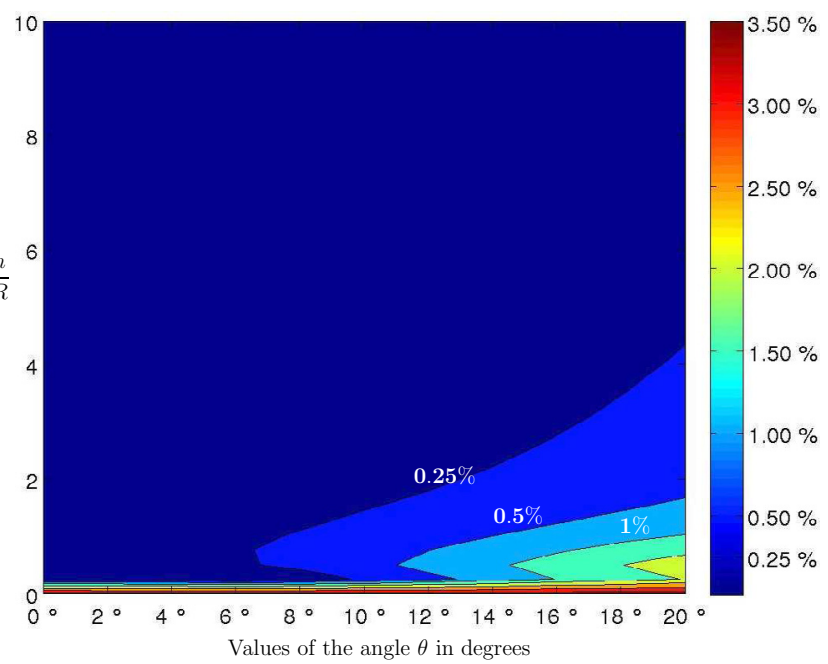

Figure 11: The error $\varepsilon_{\mathrm{inc}}=\left|\frac{K_{R, \text { inc }}^{\mathrm{app}}-K_{R, \mathrm{inc}}^{\mathrm{num}}}{K_{R, \mathrm{inc}}^{\mathrm{num}}}\right|$.

them corresponding to a set of values for $h$ and $\varphi$ given in the form of a grid of points with step-sizes $\delta \varphi$ and $\delta h$ (see Fig. 3)

$$
\varphi \in[0,10], \quad h / R_{-} \in[0,10] \quad \text { with steps } \delta \varphi=0.1 \text { and } \delta h / R_{-}=0.2 .
$$

The values of $\varphi$ and $\delta \varphi$ are given in degrees. For these axi-symmetric cases, the discrete problem to be solved is of small-size. It was hence possible to carry out the computations related to these configurations on a laptop. The geometries for $\varphi=0$ corresponds to the cylindrical case. The results for a conical perforation are collected in Figure 10.

For tilted perforations, the various configurations are similarly described by means of a grid of points $\theta$ and $h$ given by

$$
\theta \in[0,10], \quad h / R \in[0,10] \quad \text { with steps } \delta \theta=0.1 \text { and } \delta h / R=0.5 .
$$

Here too, the values of $\theta$ and $\delta \theta$ are given in degrees. The geometry is no more axi-symmetric. A three dimensional solver was required to perform the computations. To lower the discretization error at a level where it has no significant incidence on the result, we have used very refined meshes. The largest computations involved $N_{\text {dof }}:=1.5 \times 10^{5}$ degrees of freedom requiring to solve square dense linear systems of $N_{\text {dof }} \times N_{\text {dof }}$ unknowns. Such large sizes for the numerical problems have been chosen to reduce the discretization error to a negligible level. Tests on similar cases with an explicit analytical solution have shown that this error is less than $2 \%$ o (see Appendix 5.3). The computations were carried out on high performance platform with 400 cores. They took 12 hours of elapsed CPU time. The numerical results for a tilted perforation are collected in Figure 11.

Comparing these figures with Figures 8 and 9, we remark that, for large values of $h$, inequalities (5.2) predict too large errors. More precisely, the error is below $1 \%$ for $h / R>1$. This clearly shows that the proposed approximation of the Rayleigh conductivity can advantageously be used instead of the direct computation.

In Figures 12 and 13 we compute the barycentric coefficients $\lambda_{*} \in[0,1]$ of $K_{R, *}$ with respect to $K_{R, *}^{ \pm}$

$$
K_{R, \text { con }}=\left(1-\lambda_{\text {con }}\right) K_{R, \text { con }}^{-}+\lambda_{\text {con }} K_{R, \text { con }}^{+} \quad \text { and } \quad K_{R, \text { inc }}=\left(1-\lambda_{\text {inc }}\right) K_{R, \text { inc }}^{-}+\lambda_{\text {inc }} K_{R, \text { inc }}^{+} .
$$

The best approximation of $K_{R}$ is $K_{R}^{-}$for $\lambda \in[0,1 / 4], K_{R}^{\text {app }}$ for $\lambda \in[1 / 4,3 / 4]$, and $K_{R}^{+}$for $\lambda \in[3 / 4,1]$. It can be observed that for small ratio of $h / R$, the best approximation of $K_{R}$ is given by $K_{R}^{+}$, whereas for larger $h / R$, 


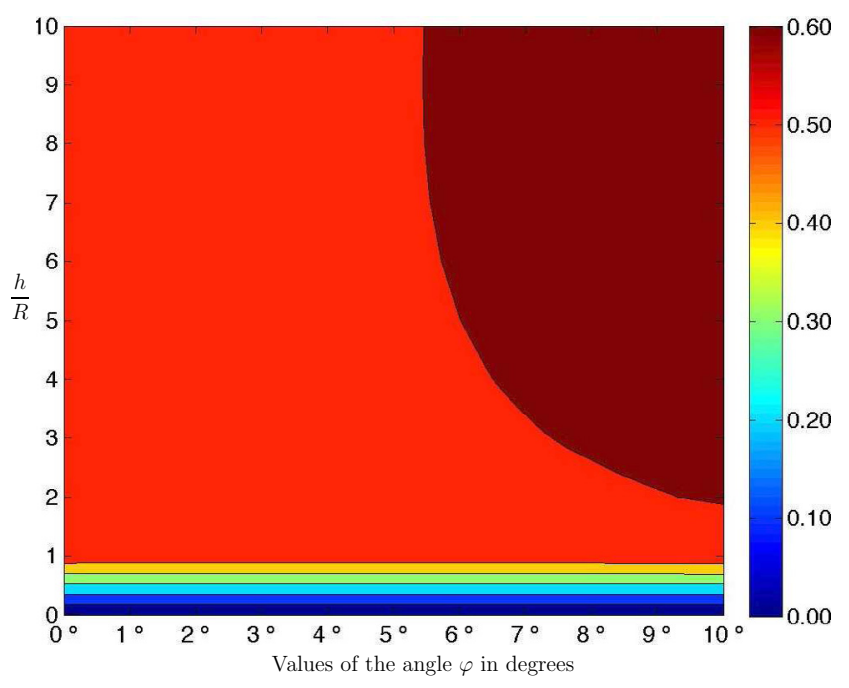

Figure 12: The barycentric coordinate $\lambda_{\text {con }}$.

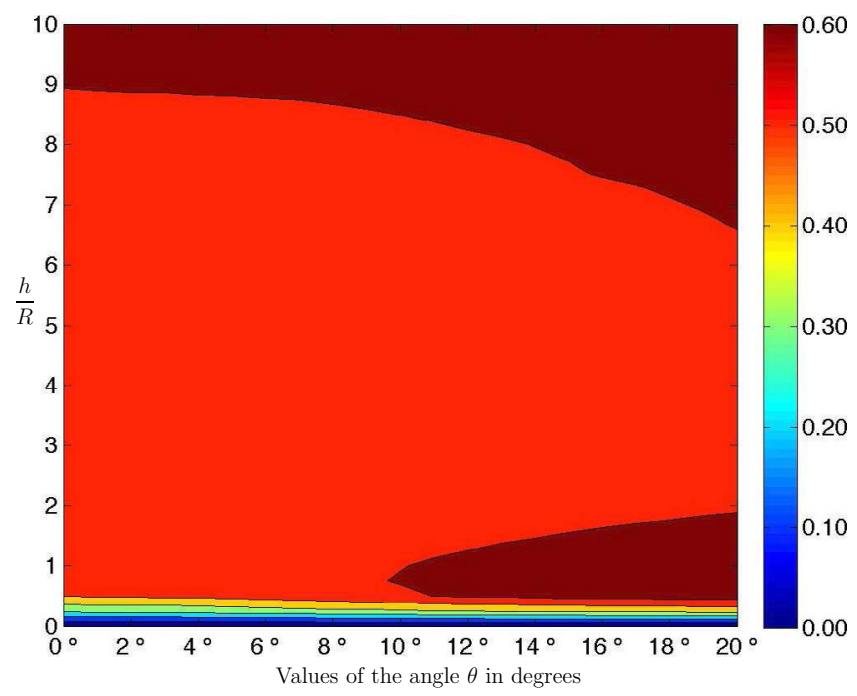

Figure 13: The barycentric coordinate $\lambda_{\text {cyl }}$.

it is given by $K_{R}^{\text {app }}$. These maps also indicate that the approximation defined in (5.1) is very accurate in most configurations.

Acknowledgements. This work was supported by the French National Research Agency under grant no. ANR-08-SYSC-001. We would like to thank the CEA-CCRT (Centre de calcul pour la recherche et la technologie) for providing us with the possibility of using the Titane computer.

\section{APPENDiX A. EXPLiCIT ANALYTICAL EXPRESSIONS FOR SOME INTEGRALS}

Let us recall that $w_{R}, t_{R}, z_{R}$, defined in (4.3), (4.12) and (4.7), are given by

$$
f_{R}(\mathbf{x})=\frac{1}{2 \pi} \int_{\mathrm{B}_{R}} \rho_{f_{R}}(\mathbf{y}) \frac{\mathrm{d} s_{\mathbf{y}}}{|\mathbf{x}-\mathbf{y}|} \quad \text { for } x_{3} \neq 0,
$$

with $f_{R}=w_{R}, t_{R}$ or $z_{R}$ and the single layer potential $\rho_{f_{R}}$ defined on $\mathrm{B}_{R}=\left\{\left(y_{1}, y_{2}, y_{3}\right) \in \mathbb{R}^{3} \mid y_{1}^{2}+y_{2}^{2}<\right.$ $R^{2}$ and $\left.y_{3}=0\right\}$ by

$$
\rho_{w_{R}}(\mathbf{y})=\frac{1}{\pi} \frac{1}{\sqrt{R^{2}-|\mathbf{y}|^{2}}}, \quad \rho_{t_{R}}(\mathbf{y})=\frac{4}{\pi} \frac{y_{1}}{\sqrt{R^{2}-|\mathbf{y}|^{2}}}, \quad \text { and } \quad \rho_{z_{R}}(\mathbf{y})=-1 .
$$

The following lemma collects three of the integrals involved in the bounds of the Rayleigh conductivity.

Lemma A.1. The following formulas hold true

$$
\begin{aligned}
& \text { (i) } \int_{x_{3}>0}\left|\nabla w_{R}(\mathbf{x})\right|^{2} \mathrm{~d} \mathbf{x}=\int_{x_{3}<0}\left|\nabla w_{R}(\mathbf{x})\right|^{2} \mathrm{~d} \mathbf{x}=R, \\
& \text { (ii) } \int_{x_{3}>0}\left|\nabla t_{R}(\mathbf{x})\right|^{2} \mathrm{~d} \mathbf{x}=\int_{x_{3}<0}\left|\nabla t_{R}(\mathbf{x})\right|^{2} \mathrm{~d} \mathbf{x}=8 R^{3} / 3, \\
& \text { (iii) } \int_{x_{3}>0}\left|\nabla z_{R}(\mathbf{x})\right|^{2} \mathrm{~d} \mathbf{x}=\int_{x_{3}<0}\left|\nabla z_{R}(\mathbf{x})\right|^{2} \mathrm{~d} \mathbf{x}=8 R^{3} / 3 .
\end{aligned}
$$


Proof. For $f_{R}=w_{R}, z_{R}$ or $t_{R}$, we have $f_{R}\left(x_{1}, x_{2},-x_{3}\right)=f_{R}\left(x_{1}, x_{2}, x_{3}\right)$, leading to

$$
I_{f_{R}}=\int_{x_{3}<0}\left|\nabla f_{R}(\mathbf{x})\right|^{2} \mathrm{~d} \mathbf{x}=\int_{x_{3}>0}\left|\nabla f_{R}(\mathbf{x})\right|^{2} \mathrm{~d} \mathbf{x} .
$$

Since $f_{R} \in \mathrm{BL}\left(\left\{x_{3}>0\right\}\right)$ and $\Delta f_{R}=0$, we get, from Green's formula and the integral representations of the solution to the related boundary value problems [28]

$$
I_{f_{R}}=\int_{x_{3}>0}\left|\nabla f_{R}(\mathbf{x})\right|^{2} \mathrm{~d} \mathbf{x}=\int_{x_{3}=0} \partial_{x_{3}} f_{R}(\mathbf{x}) f_{R}(\mathbf{x}) \mathrm{d} s_{\mathbf{x}}=\int_{\mathrm{B}_{R}} \rho_{f_{R}}(\mathbf{x}) f_{R}(\mathbf{x}) \mathrm{d} s_{\mathbf{x}} .
$$

(i) From (4.3), we set $w_{R}=1 / 2$ on $\mathrm{B}_{R}$. This leads to

$$
I_{w_{R}}=\int_{\mathrm{B}_{R}} \frac{1}{\pi \sqrt{R^{2}-|\mathbf{y}|^{2}}} \frac{1}{2} \mathrm{~d} s_{\mathbf{y}}=\int_{0}^{R} \frac{\rho \mathrm{d} \rho}{\sqrt{R^{2}-\rho^{2}}}=\left[-\sqrt{R^{2}-\rho^{2}}\right]_{0}^{R}=R .
$$

(ii) Similarly, from (4.12), we have $t_{R}(\mathbf{x})=x_{1}$ on $\mathrm{B}_{R}$. In spherical coordinates, integral (A.1) takes the form, with $y_{1}=\rho \cos \widehat{\theta}, y_{2}=\rho \sin \widehat{\theta} \cos \widehat{\varphi}$, and $y_{3}=\rho \sin \widehat{\theta} \sin \widehat{\varphi}$,

$$
\begin{aligned}
I_{t_{R}} & =\int_{\mathrm{B}_{R}} \frac{4}{\pi} \frac{y_{1}}{\sqrt{R^{2}-|\mathbf{y}|^{2}}} y_{1} \mathrm{~d} s_{\mathbf{y}}=\frac{4}{\pi} \int_{0}^{R} \int_{0}^{2 \pi} \frac{\rho \cos \widehat{\theta}}{\sqrt{R^{2}-\rho^{2}}} \rho \cos \widehat{\theta} \rho \mathrm{d} \rho d \widehat{\theta} \\
& =\frac{4}{\pi} \int_{0}^{R} \frac{\rho^{3}}{\sqrt{R^{2}-\rho^{2}}} \mathrm{~d} \rho \int_{0}^{2 \pi} \cos ^{2} \widehat{\theta} d \widehat{\theta}=-4\left[\sqrt{R^{2}-\rho^{2}}\left(\rho^{2}+2 R^{2}\right) / 3\right]_{\rho=0}^{R}=8 R^{3} / 3 .
\end{aligned}
$$

(iii) In this case, we do not know explicitly the value of $z_{R}$ on $\mathrm{B}_{R}$. However, $I_{z_{R}}$ is given by

$$
I_{z_{R}}=\frac{1}{2 \pi} \int_{\mathrm{B}_{R}} \int_{\mathrm{B}_{R}} \frac{\mathrm{d} s_{\mathbf{y}} \mathrm{d} s_{\mathbf{x}}}{|\mathbf{y}-\mathbf{x}|}=\frac{R^{3}}{2 \pi} \int_{B_{1}} \int_{B_{1}} \frac{\mathrm{d} s_{\mathbf{y}} \mathrm{d} s_{\mathbf{x}}}{|\mathbf{y}-\mathbf{x}|} .
$$

To evaluate this integral, we use Copson's formula [5]

$$
\int_{B_{1}} \frac{\mathrm{d} s_{\mathbf{y}}}{|\mathbf{x}-\mathbf{y}|}=4 \int_{\rho=0}^{1} \int_{t=0}^{\min (\rho, r)} \frac{\rho \mathrm{d} \rho \mathrm{d} t}{\sqrt{\left(\rho^{2}-t^{2}\right)\left(r^{2}-t^{2}\right)}} .
$$

Separating the cases $r<\rho$ and $\rho<r$, we are led to

$$
\begin{aligned}
I_{z_{R}} & =\frac{2 R^{3}}{\pi} \int_{B_{1}}\left(\int_{\rho=0}^{1} \int_{t=0}^{\min (\rho, r)} \frac{\rho \mathrm{d} \rho \mathrm{d} t}{\sqrt{\left(\rho^{2}-t^{2}\right)\left(r^{2}-t^{2}\right)}}\right) \mathrm{d} s_{\mathbf{x}} \\
& =4 R^{3} \int_{r=0}^{1} \int_{\rho=0}^{1} \int_{0}^{\min (\rho, r)} \frac{r \rho \mathrm{d} r \mathrm{~d} \rho \mathrm{d} t}{\sqrt{\left(\rho^{2}-t^{2}\right)\left(r^{2}-t^{2}\right)}} \\
& =4 R^{3} \int_{r=0}^{1} \int_{\rho=r}^{1} \int_{t=0}^{r} \frac{r \rho \mathrm{d} r \mathrm{~d} \rho \mathrm{d} t}{\sqrt{\left(\rho^{2}-t^{2}\right)\left(r^{2}-t^{2}\right)}}+4 R^{3} \int_{r=0}^{1} \int_{\rho=0}^{r} \int_{t=0}^{\rho} \frac{r \rho \mathrm{d} r \mathrm{~d} \rho \mathrm{d} t}{\sqrt{\left(\rho^{2}-t^{2}\right)\left(r^{2}-t^{2}\right)}} .
\end{aligned}
$$

Taking into account symmetry and commuting the integrals, we get

$$
I_{z_{R}}=8 R^{3} \int_{r=0}^{1} \int_{\rho=0}^{1} \int_{t=0}^{\rho} \frac{r \rho \mathrm{d} r \mathrm{~d} \rho \mathrm{d} t}{\sqrt{\left(\rho^{2}-t^{2}\right)\left(r^{2}-t^{2}\right)}}=8 R^{3} \int_{t=0}^{1} \int_{\rho=t}^{r} \int_{r=\rho}^{1} \frac{r \rho \mathrm{d} r \mathrm{~d} \rho \mathrm{d} t}{\sqrt{\left(\rho^{2}-t^{2}\right)\left(r^{2}-t^{2}\right)}} .
$$

Evaluating successively the integrals, we obtain

$$
\begin{aligned}
I_{z_{R}} & =8 R^{3} \int_{t=0}^{1} \int_{\rho=t}^{1}\left[\frac{\sqrt{r^{2}-t^{2}}}{\sqrt{\rho^{2}-t^{2}}}\right]_{r=\rho}^{1} \rho \mathrm{d} \rho \mathrm{d} t=8 R^{3} \int_{t=0}^{1} \int_{\rho=t}^{r}\left(\frac{\sqrt{1-t^{2}}}{\sqrt{\rho^{2}-t^{2}}}-1\right) \rho \mathrm{d} \rho \mathrm{d} t \\
& =8 R^{3} \int_{t=0}^{1}\left[\sqrt{1-t^{2}} \sqrt{\rho^{2}-t^{2}}-\rho^{2} / 2\right]_{\rho=t}^{1} \mathrm{~d} t=8 R^{3} \int_{t=0}^{1}\left(1-t^{2}\right) / 2 \mathrm{~d} t=8 R^{3} / 3 .
\end{aligned}
$$




\section{Appendix B. Direct numerical Determination of the Rayleigh Conductivity}

The numerical determination of the Rayleigh conductivity is carried out by a boundary integral method ( $c f$. , e.g., $[13,20,28])$. Here we adapt the so-called direct approach (see, e.g., [28]). The unknowns of the system to be solved will be the two interior traces, respectively denoted by $u$ and $\sigma=\partial_{\mathbf{n}} u$, of the solutions of problem (1.7) on the boundary $\partial \Omega=\Sigma \cup \Gamma_{+} \cup \Gamma_{-}$of the perforation $\Omega$. Recall that here $\mathbf{n}$ indicates the unit normal on $\partial \Omega$ directed outwards $\Omega$. The formulation relies upon the following representation formula

$$
\begin{gathered}
u(\mathbf{x})=V \sigma(\mathbf{x})+N u(\mathbf{x}), \quad \mathbf{x} \in \Omega, \\
\begin{cases}u(\mathbf{x})=1 / 2-2 V_{+} \sigma(\mathbf{x}) & \text { for } x_{3}>s_{+}, \\
u(\mathbf{x})=-1 / 2-2 V_{-} \sigma(\mathbf{x}) & \text { for } x_{3}<s_{-},\end{cases}
\end{gathered}
$$

where $V, N, V_{ \pm}$are the following integral operators:

$$
\left\{\begin{array}{l}
V \sigma(\mathbf{x})=\int_{\partial \Omega} \frac{1}{4 \pi|\mathbf{x}-\mathbf{y}|} \sigma(\mathbf{y}) \mathrm{d} s_{\mathbf{y}} \\
N u(\mathbf{x})=-\int_{\partial \Omega} \partial_{\mathbf{n}_{\mathbf{y}}} \frac{1}{4 \pi|\mathbf{x}-\mathbf{y}|} u(\mathbf{y}) \mathrm{d} s_{\mathbf{y}} \\
V_{ \pm} \sigma(\mathbf{x})=\frac{1}{2} \int_{\Gamma_{ \pm}}\left(\frac{1}{4 \pi|\mathbf{x}-\mathbf{y}|}+\frac{1}{4 \pi\left|\mathbf{x}_{ \pm}^{*}-\mathbf{y}\right|}\right) \sigma(\mathbf{y}) \mathrm{d} s_{\mathbf{y}}
\end{array}\right.
$$

where $\mathbf{x}_{ \pm}^{*}$ is the symmetrical point of $\mathbf{x}$ relatively to the plane $\left\{x_{3}=s_{ \pm}\right\}$. Formula (B.1) is the very classical integral representation of harmonic functions in the interior of a bounded domain (cf., e.g., [13], Eq. (1.1.7), p. 3 and Rem. 1.5). Formula (B.2) and (B.3) is the usual expression of the solution $u$ in the half-spaces $\left\{x_{3}>s_{+}\right\}$ (resp. $\left\{x_{3}<s_{-}\right\}$) based upon the corresponding Green function

$$
G_{\infty}^{ \pm}(\mathbf{x}, \mathbf{y})=\frac{1}{4 \pi|\mathbf{x}-\mathbf{y}|}+\frac{1}{4 \pi\left|\mathbf{x}_{ \pm}^{*}-\mathbf{y}\right|}
$$

(cf., e.g., [12], p. 355). Taking into account that $\left|\mathbf{x}_{ \pm}^{*}-\mathbf{y}\right|=|\mathbf{x}-\mathbf{y}|$ for $\mathbf{y} \in \Gamma_{ \pm}$, we get

$$
V_{ \pm} \sigma(\mathbf{x})=\int_{\Gamma_{ \pm}} \frac{1}{4 \pi|\mathbf{x}-\mathbf{y}|} \sigma(\mathbf{y}) \mathrm{d} s_{\mathbf{y}} .
$$

The direct method is based on the following trace formula

$$
\begin{cases}u(\mathbf{x})=1 / 2 u(\mathbf{x})+V \sigma(\mathbf{x})+N u(\mathbf{x}) & \mathbf{x} \in \partial \Omega \\ \sigma(\mathbf{x})=1 / 2 \sigma(\mathbf{x})-N^{T} \sigma(\mathbf{x})+D u(\mathbf{x}) & \mathbf{x} \in \partial \Omega\end{cases}
$$

where $N^{T}$ is the adjoint of the integral operator $N$ and $D$ is the integral operator corresponding to a hypersingular kernel which can be expressed variationally as (cf., e.g., [13] p. 5)

$$
\left\langle D \sigma, \sigma^{\prime}\right\rangle_{\partial \Omega}=\int_{\partial \Omega \times \partial \Omega} \frac{1}{4 \pi|\mathbf{x}-\mathbf{y}|}\left(\mathbf{n}_{\mathbf{y}} \times \nabla_{\mathbf{y}} \sigma(\mathbf{y})\right) \cdot\left(\mathbf{n}_{\mathbf{x}} \times \nabla_{\mathbf{x}} \sigma^{\prime}(\mathbf{x})\right) \mathrm{d} s_{\mathbf{x}} \mathrm{d} s_{\mathbf{y}},
$$

for sufficiently smooth $\sigma$ and $\sigma^{\prime}$, with $\langle\cdot, \cdot\rangle_{\partial \Omega}$ the duality brackets between the trace space $H^{1 / 2}(\partial \Omega)$ and its dual $H^{-1 / 2}(\partial \Omega)$. Clearly, the boundary condition on $\Sigma$ yields

$$
\sigma(\mathbf{x})=0 \text { on } \Sigma \text {. }
$$

The adaptation of the direct method in the present context consists in considering the relations linking the two traces on $\Gamma_{+}$and $\Gamma_{-}$expressed by equations (B.2) and (B.3) and additional constraints on the remaining part of $\partial \Omega$

$$
u(\mathbf{x})+2 V_{ \pm} \sigma(\mathbf{x})= \pm 1 / 2 \quad \text { for } \mathbf{x} \in \Gamma_{ \pm} .
$$


Let $f$ be a function in $H^{1 / 2}(\partial \Omega)$ equal to $1 / 4$ on $\Gamma_{+}$and $-1 / 4$ on $\Gamma_{-}$. Equation (B.13) can thus be rewritten as

$$
1 / 2 u(\mathbf{x})+V_{ \pm} \sigma(\mathbf{x})=f(\mathbf{x}) \quad \text { for } \mathbf{x} \in \Gamma_{ \pm} .
$$

The constraint (B.12) is taken into account explicitely whereas constraints (B.14) are handled by means of a Lagrange multiplier. More precisely, starting from (B.9) and (B.14), we can write

$$
\left\langle V \sigma, \sigma^{\prime}\right\rangle_{\partial \Omega}+\left\langle N u, \sigma^{\prime}\right\rangle_{\partial \Omega}=\left\langle 1 / 2 u, \sigma^{\prime}\right\rangle_{\partial \Omega}=-\left\langle V_{+} \sigma, \sigma^{\prime}\right\rangle_{\Gamma_{+}}-\left\langle V_{-} \sigma, \sigma^{\prime}\right\rangle_{\Gamma_{-}}+\left\langle f, \sigma^{\prime}\right\rangle_{\partial \Omega}
$$

for all $\sigma^{\prime} \in H^{-1 / 2}(\partial \Omega)$ with $\sigma^{\prime}(\mathbf{x})=0$ on $\Sigma$. In the same way, we can write equation (B.10) in the form

$$
\left\langle u^{\prime}, N^{T} \sigma\right\rangle_{\partial \Omega}-\left\langle u^{\prime}, D u\right\rangle_{\partial \Omega}=-\left\langle 1 / 2 u^{\prime}, \sigma\right\rangle_{\partial \Omega},
$$

for all $u^{\prime} \in H^{1 / 2}(\partial \Omega)$. Finally equation (B.14), can be written as

$$
\left\langle V_{+} \sigma, \ell^{\prime}\right\rangle_{\Gamma_{+}}+\left\langle V_{-} \sigma, \ell^{\prime}\right\rangle_{\Gamma_{-}}+\left\langle 1 / 2 u, \ell^{\prime}\right\rangle_{\partial \Omega}=\left\langle f, \ell^{\prime}\right\rangle_{\partial \Omega}
$$

for all $\ell^{\prime} \in H^{-1 / 2}(\partial \Omega)$ with $\ell^{\prime}(\mathbf{x})=0$ on $\Sigma$. Noting that $\left\langle V_{+} \sigma, \sigma^{\prime}\right\rangle_{\Gamma_{+}}+\left\langle V_{-} \sigma, \sigma^{\prime}\right\rangle_{\Gamma_{-}}=\left\langle V \sigma, \sigma^{\prime}\right\rangle_{\partial \Omega}$ and substituting $\ell$ for $\sigma$ in the respective right-hand sides of equations (B.15) and (B.16), we readily arrive to the following formulation in the form of a saddle-point problem

Find $\{(u, \sigma), \ell\} \in W \times L$ such that for all $\left\{\left(u^{\prime}, \sigma^{\prime}\right), \ell^{\prime}\right\} \in W \times L$

$$
\begin{cases}a\left((u, \sigma),\left(u^{\prime}, \sigma^{\prime}\right)\right)+b\left(\ell,\left(u^{\prime}, \sigma^{\prime}\right)\right) & =\left\langle f, \sigma^{\prime}\right\rangle_{\partial \Omega}, \\ b\left(\ell^{\prime},(u, \sigma)\right) & =\left\langle f, \ell^{\prime}\right\rangle_{\partial \Omega}\end{cases}
$$

with

$$
\left\{\begin{aligned}
a\left((u, \sigma),\left(u^{\prime}, \ell^{\prime}\right)\right) & =\left\langle V \sigma, \sigma^{\prime}\right\rangle_{\partial \Omega}+\left\langle N u, \sigma^{\prime}\right\rangle_{\partial \Omega}+\left\langle N u^{\prime}, \sigma\right\rangle_{\partial \Omega}-\left\langle D u, u^{\prime}\right\rangle_{\partial \Omega}, \\
b\left(\ell^{\prime},(u, \sigma)\right) & =\left\langle V \sigma+1 / 2 u, \ell^{\prime}\right\rangle_{\partial \Omega}
\end{aligned}\right.
$$

and

$$
L=\left\{\sigma \in H^{-1 / 2}(\partial \Omega) ; \sigma_{\left.\right|_{\Sigma}}=0\right\} \quad \text { and } \quad W=H^{1 / 2}(\partial \Omega) \times L .
$$

System (B.18) is discretized using a triangular mesh of $\partial \Omega$ and approximating any involved function by a $\mathbb{P}_{1^{-}}$ continuous boundary element method (cf., e.g., [3] and the references therein). It is worth mentioning that $L$ is actually approximated by a finite element method built on respectively $\Gamma_{+}$and $\Gamma_{-}$with the nodal values equal to 0 on the respective boundary curves of $\Gamma_{+}$and $\Gamma_{-}$. The accuracy of the approach has been validated from the cases of circular and elliptical perforations in an infinitely thin plate for which the exact analytically value of the corresponding Rayleigh conductivities are known. In this case, the relative errors were less than $2 \%$.

\section{REFERENCES}

[1] M. Alster, Improved calculation of resonant frequencies of Helmholtz resonators. J. Sound Vibr. 24 (1972) 63-85.

[2] C. Amrouche, V. Girault and J. Giroire, Dirichlet and Neumann exterior problems for the n-dimensional Laplace operator an approach in weighted sobolev spaces. J. Math. Pures Appl. 76 (1997) 55-81.

[3] A. Bendali and M. Fares, Boundary integral equations methods in acoustics. Computer Methods for Acoustics Problems, Chapter 1. Edited by F. Magoules. Saxe-Coburg Publications, Kippen, Stirlingshire, Scotland (2008) 1-36.

[4] R.C. Chanaud, Effects of geometry on the resonance frequency of Helmholtz resonators, part II. J. Sound Vibr. 204 (1997) 829-834.

[5] E.T. Copson, On the problem of the electrified disc. Proc. Edinburgh Math. Soc. (Ser. 2) 8 (1947) 14-19.

[6] R. Courant and D. Hilbert, Methods of Mathematical Physics. Vol. I. Interscience Publishers, Inc., New York, N.Y. (1953).

[7] J. Deny and J.L. Lions. Les espaces du type de Beppo Levi. Ann. Inst. Fourier 5 (1953) 54.

[8] B. Enquist and A. Majda, Absorbing boundary conditions for the numerical simulation of wave. Math. Comput. 31 (1977) $629-651$. 
[9] J. Giroire, Étude de quelques problèmes aux limites extérieurs et résolution par équations intégrales. Ph.D. Thesis. Paris VI (1987).

[10] M.S. Howe, On the theory of unsteady high Reynolds number flow through a circular aperture. Proc. Roy. Soc. London A. Math. Phys. Sci. 366 (1979) 205.

[11] M.S. Howe, Influence of wall thickness on Rayleigh conductivity and flow-induced aperture tones. J. Fluids Struct. 11 (1997) 351-366.

[12] M.S. Howe, Acoustics of fluid-structure interactions. Cambridge University Press (1998).

[13] G. C. Hsiao and W.L. Wendland, Boundary Integral Equations. Springer, Berlin-Heidelberg (2008).

[14] U. Ingard, On the theory and design of acoustic resonators. J. Acoust. Soc. America 25 (1953) 1037-1061.

[15] J.B. Keller and D. Givoli, Exact non-reflecting boundary conditions. J. Comput. Phys. 82 (1989) 172-192.

[16] E. Kerschen, A. Cain and G. Raman, Analytical Modeling of Helmholtz Resonator Based Powered Resonance Tubes, in 2nd AIAA Flow Control Conference, AIAA Paper 2004-2691 (2004).

[17] D.G. Luenberger, Optimization by vector space methods. Wiley-Interscience (1997).

[18] C. Macaskill and E.O. Tuck, Evaluation of the acoustic impedance of a screen. J. Australian Math. Soc. Ser. B Appl. Math. 20 (1977) 46-61.

[19] C. Malmary, Étude théorique et expérimentale de l'impédance acoustique de matériaux en présence d'un écoulement d'air tangentiel. Ph.D. thesis. University du Maine (2000).

[20] William McLean, Strongly Elliptic Systems and Boundary Integral Equations. Cambridge University Press, Cambridge, UK, and New York, USA (2000).

[21] T.H. Melling, The acoustic impendance of perforates at medium and high sound pressure levels. J. Sound Vibr. 29 (1973) $1-65$.

[22] S. Mendez and J.D. Eldredge, Acoustic modeling of perforated plates with bias flow for Large-Eddy Simulations. J. Comput. Phys. 228 (2009) 4757-4772.

[23] J. Mohring, Helmholtz resonators with large aperture. Acta Acoust. United Acoust. 85 (1999) 751-763.

[24] C.L. Morfey, Acoustic properties of openings at low frequencies. J. Sound Vibr. 9 (1969) 357-366.

[25] R.L. Panton and J.M. Miller, Resonant frequencies of cylindrical Helmholtz resonators. J. Acoust. Soc. America 57 (1975) $1533-1535$.

[26] J.W.S. Rayleigh, The Theory of Sound, vols. 1 and 2. Dover Publications, New York (1945).

[27] J.E. Roberts and J.-M. Thomas, Mixed and hybrid methods, in Handbook of numerical analysis. Vol. 2. Elsevier Science Publishers (1991).

[28] S. Sauter and C. Schwab, Boundary Element Methods, vol. 39 of Springer Series in Computational Mathematics. Springer, Heidelberg (2010).

[29] I.N. Sneddon, Mixed boundary value problems in potential theory. North-Holland Pub. Co. (1966).

[30] E.O. Tuck, Matching problems involving flow through small holes. Adv. Appl. Mech. 15 (1975) 89-158. 